\title{
Global regularity for an inviscid three-dimensional slow limiting ocean dynamics model
}

\author{
Chongsheng Cao, Aseel Farhat, and Edriss S. Titi \\ Dedicated to Professor Marshall Slemrod on the occasion of his $70^{\text {th }}$ \\ birthday
}

\begin{abstract}
We establish, for smooth enough initial data, the global well-posedness (existence, uniqueness and continuous dependence on initial data) of solutions, for an inviscid three-dimensional slow limiting ocean dynamics model. This model was derived as a strong rotation limit of the rotating and stratified Boussinesq equations with periodic boundary conditions. To establish our results, we utilize the tools developed for investigating the two-dimensional incompressible Euler equations and linear transport equations. Using a weaker formulation of the model, we also show the global existence and uniqueness of solutions, for less regular initial data.
\end{abstract}

MSC SUBJECT CLASSIFICATIONS: 35Q35, 76B03, 86A10.

KEYWORDS AND PHRASES: Three-dimensional Boussinesq equations, slowdynamics, ocean model, global regularity.

\section{Introduction}

The questions of global well-posedness of the three-dimesional Navier-Stokes and Euler equations, as well as the three-dimensional Boussinesq equations of incompressible flows, are considered to be among the most challenging mathematical problems in applied analysis. In the context of the atmosphere and the ocean circulation dynamics, geophysicists take advantage of the fast rotation effect to simplify the Boussinesq equations. The Taylor-Proudman theorem [16] suggests that the fluid velocity will be uniform along any line parallel to the axis of rotation and that the fluid motion takes place in tall columnar structures. To determine the strength of the rotation (Coriolis force), Rossby number (Ro) is often used. Rossby number (Ro) is a dimensionless number that measures that ratio between a characteristic value of the inertial force term to the Coriolis force. Thus, the smaller the Rossby number is, the stronger the rotation effect is. In a recent work [17], the 
authors explored the fast rotation limit (Rossby number $R o \rightarrow 0$ ) of the rotating stratified Boussinesq equations to derive a system for the "slow" dynamics. Subject to periodic boundary conditions in $\mathbb{T}^{3}=[0, L]^{3}$, the viscous version of the slow limiting dynamics model, that was introduced in [17], is given by:

$$
\begin{aligned}
\frac{\partial \mathbf{u}_{h}}{\partial t}+\left(\mathbf{u}_{h} \cdot \nabla_{h}\right) \mathbf{u}_{h}+\nabla_{h} p & =\frac{1}{R e} \Delta_{h} \mathbf{u}_{h} \\
\frac{\partial w}{\partial t}+\left(\mathbf{u}_{h} \cdot \nabla_{h}\right) w & =\frac{1}{R e} \Delta_{h} w-\frac{1}{F r}\langle\rho\rangle_{z} \\
\frac{\partial \rho}{\partial t}+(\mathbf{u} \cdot \nabla) \rho-\frac{1}{F r} w & =\frac{1}{\operatorname{RePr}} \Delta \rho \\
\nabla_{h} \cdot \mathbf{u}_{h}=0, \quad \nabla \cdot \mathbf{u} & =0
\end{aligned}
$$

where $\mathbf{u}=\left(\mathbf{u}_{h}, w\right)=\left(\mathbf{u}_{h}(t ; x, y), w(t ; x, y)\right)$ is the velocity vector field, $p=$ $p(t ; x, y)$ is the pressure, $\rho=\rho(t ; x, y, z)$ is the density fluctuation, $\nabla_{h}=$ $\left(\frac{\partial}{\partial x}, \frac{\partial}{\partial y}\right), \Delta_{h}=\frac{\partial^{2}}{\partial x^{2}}+\frac{\partial^{2}}{\partial y^{2}}, \Delta=\frac{\partial^{2}}{\partial x^{2}}+\frac{\partial^{2}}{\partial y^{2}}+\frac{\partial^{2}}{\partial z^{2}}, R e$ is the Reynolds number (the ratio of inertial forces to viscous force), $\mathrm{Fr}$ is the Froude number (the ratio of a characteristic velocity to a gravitational wave velocity) and $\mathrm{Pr}$ is the Prandtl number (the ratio of momentum diffusivity to thermal diffusivity). Also, $\langle\rho\rangle_{z}$ is the density average in the vertical direction defined by

$$
\langle\rho\rangle_{z}(t ; x, y):=\frac{1}{L} \int_{[0, L]} \rho(t ; x, y, z) d z .
$$

The derivation of this model is based on the assumption that the solution evolves only on the slow advective time scale. For this reason the system was called a slow limiting dynamics model, even though it was derived in the limit of fast rotation. If the initial data contains inertial waves, then the model has to be modified to take into account the fast inertial waves.

In the slow limiting dynamics model (1.1), the horizontal component of the velocity $\mathbf{u}_{h}$ is governed by the $2 \mathrm{D}$ Navier-Stokes equations. Moreover, $\mathbf{u}_{h}$ evolves independently of the vertical velocity $w$ and the density fluctuation $\rho$, but it influences the dynamics of these variables through the advection terms in (1.1b) and (1.1c). The dynamics of the vertical velocity $w$ and the density fluctuation $\rho$ are strongly coupled. Interestingly, the vertical velocity $w$ evolves according to a two-dimensional forced advection-diffusion equation, (1.1b), with buoyancy force given by $\langle\rho\rangle_{z}$, the density average in the vertical direction. However, the evolution equation of the density $\rho$ in (1.1c) retains its three-dimensionality. 
In [17], numerical simulations that use low wavenumber white noise forcing in the momentum equations were produced. The flow characteristics were examined at different Rossby numbers. The formulation and the presence of large-scale columnar Taylor-Proudman flows appeared as $R o \rightarrow 0$. It was also observed that the smaller the Rossby number the larger the magnitude of the kinetic energy of the slow limiting model. Moreover, as the Rossby number decreases, the ratio of the kinetic energy of the slow limiting model to the total kinetic energy of the rotating Boussinesq system increases gradually to a constant close to 1 .

We notice that taking the $z$-average of (1.1c) implies that $\langle\rho\rangle_{z}$ satisfies the evolution equation:

$$
\frac{\partial\langle\rho\rangle_{z}}{\partial t}+\left(\mathbf{u}_{h} \cdot \nabla_{h}\right)\langle\rho\rangle_{z}=\frac{1}{F r} w+\frac{1}{\operatorname{RePr}} \Delta_{h}\langle\rho\rangle_{z} .
$$

We introduce here the inviscid version of system (1.1):

$$
\begin{aligned}
& \frac{\partial \mathbf{u}_{h}}{\partial t}+\left(\mathbf{u}_{h} \cdot \nabla_{h}\right) \mathbf{u}_{h}+\nabla_{h} p=0 \\
& \frac{\partial w}{\partial t}+\left(\mathbf{u}_{h} \cdot \nabla_{h}\right) w=-\frac{1}{F r}\langle\rho\rangle_{z} ; \quad\langle\rho\rangle_{z}\left(t ; \mathbf{x}_{h}\right):=\frac{1}{L} \int_{0}^{L} \rho(t ; \mathbf{x}) d z \\
& \frac{\partial\langle\rho\rangle_{z}}{\partial t}+\left(\mathbf{u}_{h} \cdot \nabla_{h}\right)\langle\rho\rangle_{z}=\frac{1}{F r} w \\
& \frac{\partial \rho}{\partial t}+\left(\mathbf{u}_{h} \cdot \nabla_{h}\right) \rho+w \frac{\partial \rho}{\partial z}=\frac{1}{F r} w \\
& \nabla_{h} \cdot \mathbf{u}_{h}=0, \quad \frac{\partial w}{\partial z}=0, \\
& \mathbf{u}_{h}\left(0 ; \mathbf{x}_{h}\right)=\mathbf{u}_{h}^{0}\left(\mathbf{x}_{h}\right), \quad w\left(0 ; \mathbf{x}_{h}\right)=w^{0}\left(\mathbf{x}_{h}\right), \quad \rho(0 ; \mathbf{x})=\rho^{0}(\mathbf{x})
\end{aligned}
$$

Denote by $\mathbb{T}^{d}$ the $L$-period box $[0, L]^{d}$. In this work, we will establish the global well-posedness of strong solutions, for smooth enough initial data, and the global existence and uniqueness of weak solutions, for less regular initial data, for the inviscid system (1.3) in the three-dimensional torus $\mathbb{T}^{3}$, i.e. subject to periodic boundary conditions. This paper is organized as follows. In section 2, we recall the global well-posedness of the two-dimensional incompressible Euler equations and the global existence and uniqueness of solutions for linear transport equations. In section 3, we prove the global existence and uniqueness of weak solutions of (1.3). Moreover, we prove the well-posedness (continuous dependence on initial data) of system (1.3). In section 4, we introduce a presentation of system (1.3) in vorticity formulation, and prove the global existence and uniqueness of weak solutions 
(without continuous dependence on initial data) for the system in this presentation.

\section{Preliminaries}

In this section, we introduce some preliminary material and notations which are commonly used in the mathematical study of fluids, in particular in the study of the Navier-Stokes equations (NSE) and the Euler equations.

Let $\mathcal{F}_{h}, \mathcal{F}$ be the set of all vector-valued trigonometric polynomials of zero-average with periodic domain $\mathbb{T}^{2}$ and $\mathbb{T}^{3}$, respectively. We define the spaces of smooth functions which incorporates the divergence-free and zeroaverage condition to be:

$$
\begin{gathered}
\mathcal{V}_{h}:=\left\{\varphi \in \mathcal{F}_{h}: \nabla_{h} \cdot \varphi=0 \text { and } \int_{\mathbb{T}^{2}} \varphi d x=0\right\}, \\
\mathcal{V}:=\left\{\phi \in \mathcal{F}: \nabla \cdot \phi=0 \text { and } \int_{\mathbb{T}^{3}} \phi d x=0\right\} .
\end{gathered}
$$

We denote by $L_{h}^{p}\left(\mathbb{T}^{2}\right), W_{h}^{s, p}\left(\mathbb{T}^{2}\right), H_{h}^{s}\left(\mathbb{T}^{2}\right) \equiv W_{h}^{s, 2}\left(\mathbb{T}^{2}\right)$ to be the closures of $\mathcal{V}_{h}$ in the usual Lebesgue and Sobolev spaces. Similarly, we denote by $L^{p}\left(\mathbb{T}^{3}\right)$, $W^{s, p}\left(\mathbb{T}^{3}\right), H^{s}\left(\mathbb{T}^{3}\right) \equiv W^{s, 2}\left(\mathbb{T}^{3}\right)$ to be the closures of $\mathcal{V}$ in the usual Lebesgue and Sobolev spaces, respectively.

Since we restrict ourselves to finding solutions over the three-dimensional $L$-periodic box $\mathbb{T}^{3}$, therefore, if we assume that $\int_{\mathbb{T}^{2}} \mathbf{u}_{h}^{0} d \mathbf{x}_{h}=\mathbf{0}, \int_{\mathbb{T}^{2}} w^{0} d \mathbf{x}_{h}=$ $\int_{\mathbb{T}^{3}} \rho^{0} d \mathbf{x}=0$, then integrating system (1.3) implies that

$$
\begin{aligned}
\int_{\mathbb{T}^{2}} \mathbf{u}_{h}\left(t ; \mathbf{x}_{h}\right) d \mathbf{x}_{h} & =\mathbf{0} \\
\frac{d}{d t} \int_{\mathbb{T}^{2}} w\left(t ; \mathbf{x}_{h}\right) d \mathbf{x}_{h} & =-\frac{1}{F r} \int_{\mathbb{T}^{2}}\langle\rho\rangle_{z}\left(t ; \mathbf{x}_{h}\right) d \mathbf{x}_{h}, \\
\frac{d}{d t} \int_{\mathbb{T}^{2}}\langle\rho\rangle_{z}\left(t ; \mathbf{x}_{h}\right) d \mathbf{x}_{h} & =\frac{1}{F r} \int_{\mathbb{T}^{2}} w\left(t ; \mathbf{x}_{h}\right) d \mathbf{x}_{h}, \\
\frac{d}{d t} \int_{\mathbb{T}^{3}} \rho(t ; \mathbf{x}) d \mathbf{x} & =\frac{L}{F r} \int_{\mathbb{T}^{2}} w\left(t ; \mathbf{x}_{h}\right) d \mathbf{x}_{h},
\end{aligned}
$$

for any $t>0$. This yield

$$
\frac{d}{d t}\left(\int_{\mathbb{T}^{2}} w\left(t ; \mathbf{x}_{h}\right) d \mathbf{x}_{h}+\int_{\mathbb{T}^{2}}\langle\rho\rangle_{z}\left(t ; \mathbf{x}_{h}\right) d \mathbf{x}_{h}\right)
$$




$$
\begin{aligned}
& =\frac{1}{F r}\left(\int_{\mathbb{T}^{2}} w\left(t ; \mathbf{x}_{h}\right) d \mathbf{x}_{h}-\int_{\mathbb{T}^{2}}\langle\rho\rangle_{z}\left(t ; \mathbf{x}_{h}\right) d \mathbf{x}_{h}\right), \\
\frac{d}{d t} & \left(\int_{\mathbb{T}^{2}} w\left(t ; \mathbf{x}_{h}\right) d \mathbf{x}_{h}-\int_{\mathbb{T}^{2}}\langle\rho\rangle_{z}\left(t ; \mathbf{x}_{h}\right) d \mathbf{x}_{h}\right) \\
& =\frac{-1}{F r}\left(\int_{\mathbb{T}^{2}} w\left(t ; \mathbf{x}_{h}\right) d \mathbf{x}_{h}+\int_{\mathbb{T}^{2}}\langle\rho\rangle_{z}\left(t ; \mathbf{x}_{h}\right) d \mathbf{x}_{h}\right),
\end{aligned}
$$

for any $t>0$. So, $\int_{\mathbb{T}^{2}} w\left(t ; \mathbf{x}_{h}\right) d \mathbf{x}_{h}=\int_{\mathbb{T}^{2}}\langle\rho\rangle_{z}\left(t ; \mathbf{x}_{h}\right) d \mathbf{x}_{h}=0$, for any $t \geq 0$. This yields

$$
\frac{d}{d t} \int_{\mathbb{T}^{3}} \rho(t ; \mathbf{x}) d \mathbf{x}=0,
$$

for any $t>0$. Thus, we can work in the spaces defined above consistently.

We define the inner products on $L_{h}^{2}\left(\mathbb{T}^{2}\right)$ and $H_{h}^{1}\left(\mathbb{T}^{2}\right)$, respectively, by

$$
(\mathbf{u}, \mathbf{v})_{h}=\sum_{i=1}^{2} \int_{\mathbb{T}^{2}} u^{i} v^{i} d \mathbf{x}_{h} \quad \text { and } \quad((\mathbf{u}, \mathbf{v}))_{h}=\sum_{i, j=1}^{2} \int_{\mathbb{T}^{2}} \partial_{j} u^{i} \partial_{j} v^{i} d \mathbf{x}_{h},
$$

and the associated norms $\|\mathbf{u}\|_{L_{h}^{2}\left(\mathbb{T}^{2}\right)}=(\mathbf{u}, \mathbf{u})_{h}^{1 / 2}$ and $\|\mathbf{u}\|_{H_{h}^{1}\left(\mathbb{T}^{2}\right)}=((\mathbf{u}, \mathbf{u}))_{h}^{1 / 2}$. Similarly, we define the inner products on $L^{2}\left(\mathbb{T}^{3}\right)$ and $H^{1}\left(\mathbb{T}^{3}\right)$, respectively, by

$$
(\mathbf{u}, \mathbf{v})=\sum_{i=1}^{3} \int_{\mathbb{T}^{3}} u^{i} v^{i} d \mathbf{x} \quad \text { and } \quad((\mathbf{u}, \mathbf{v}))=\sum_{i, j=1}^{3} \int_{\mathbb{T}^{3}} \partial_{j} u^{i} \partial_{j} v^{i} d \mathbf{x},
$$

and the associated norms $\|\mathbf{u}\|_{L^{2}\left(\mathbb{T}^{3}\right)}=(\mathbf{u}, \mathbf{u})^{1 / 2}$ and $\|\mathbf{u}\|_{H^{1}\left(\mathbb{T}^{3}\right)}=((\mathbf{u}, \mathbf{u}))^{1 / 2}$. (We use the same notation for both scalar and vector-valued functions and which one is meant, will be clear from context). Note that $((\cdot, \cdot))_{h}$ and $((\cdot, \cdot))$ are norms due to the Poincaré inequality, Lemma 2.2, below.

Let $Y$ be a Banach space. We denote by $L^{p}([0, T] ; Y)$ the space of (Bochner) measurable functions $t \mapsto w(t)$, where $w(t) \in Y$, for a.e. $t \in[0, T]$, such that the integral $\int_{0}^{T}\|w(t)\|_{Y}^{p} d t$ is finite.

Remark 2.1. In this paper, $C$ represents a dimensionless constant that may change from line to line.

We recall the well-known two-dimensional elliptic estimate, due to the Biot-Savart law, for $\nabla_{h} \cdot \mathbf{u}_{h}=0$ and $\nabla_{h} \times \mathbf{u}_{h}=\omega$,

$$
\left\|\mathbf{u}_{h}\right\|_{W_{h}^{1, p}\left(\mathbb{T}^{2}\right)} \leq C p\|\omega\|_{L_{h}^{p}\left(\mathbb{T}^{2}\right)}
$$


for every $p \in[2, \infty)$ (see, e.g., [18] and references therein), where $C$ is a dimensionless constant, which is independent of $p$.

Furthermore, we have the Poincaré inequality:

Lemma 2.2. [6] For all $\varphi \in H_{h}^{1}\left(\mathbb{T}^{2}\right)$ and $\phi \in H^{1}\left(\mathbb{T}^{3}\right)$, we have

$$
\|\varphi\|_{L_{h}^{2}\left(\mathbb{T}^{2}\right)} \leq C L\left\|\nabla_{h} \varphi\right\|_{L_{h}^{2}\left(\mathbb{T}^{2}\right)}
$$

and

$$
\|\phi\|_{L^{2}\left(\mathbb{T}^{3}\right)} \leq C L\|\nabla \varphi\|_{L^{2}\left(\mathbb{T}^{3}\right)}
$$

Next, we recall the global existence and uniqueness theorem, due to Yudovich, [18], for the incompressible two-dimensional Euler equations in vorticity formulations (see also $[1,12,15]$ ).

The two-dimensional Euler equations, for incompressible inviscid flows, in the periodic box $\mathbb{T}^{2}$ are

$$
\begin{array}{ll}
\frac{\partial \mathbf{u}_{h}}{\partial t}+\left(\mathbf{u}_{h} \cdot \nabla_{h}\right) \mathbf{u}_{h}+\nabla_{h} p=0, & \text { in }[0, T] \times \mathbb{T}^{2} \\
\nabla_{h} \cdot \mathbf{u}_{h}=0, & \text { in }[0, T] \times \mathbb{T}^{2} \\
\mathbf{u}_{h}\left(0 ; \mathbf{x}_{h}\right)=\mathbf{u}_{h}^{0}\left(\mathbf{x}_{h}\right), & \text { in } \mathbb{T}^{2},
\end{array}
$$

where $T>0$ is given. Here, $\mathbf{u}_{h}=\mathbf{u}_{h}(t ; x, y)$ is the velocity vector field, $p=$ $p(t ; x, y)$ is the pressure. The vorticity formulation, for the two-dimensional incompressible Euler equations is

$$
\begin{array}{ll}
\frac{\partial \omega}{\partial t}+\left(\mathbf{u}_{h} \cdot \nabla_{h}\right) \omega=0, & \text { in }[0, T] \times \mathbb{T}^{2} \\
\nabla_{h} \cdot \mathbf{u}_{h}=0, \quad \omega=\nabla_{h} \times \mathbf{u}_{h}, & \text { in }[0, T] \times \mathbb{T}^{2} \\
\omega\left(0 ; \mathbf{x}_{h}\right)=\omega^{0}\left(\mathbf{x}_{h}\right), & \text { in } \mathbb{T}^{2} .
\end{array}
$$

The velocity is determined from the vorticity by means of the two-dimensional periodic Biot-Savart law:

$$
\begin{gathered}
\mathbf{u}_{h}(x, y)=\begin{array}{rl}
K * & *
\end{array}:=\int_{\mathbb{T}^{2}} K(x-s, y-\xi) \omega(s, \xi) d s d \xi \\
K(x, y)=\nabla_{h}^{\perp} G(x, y)
\end{gathered}
$$

where $G(x, y)$ is the fundamental solution of the Poisson equation in twodimensions subject to periodic boundary conditions, the binary operation ${ }_{h}^{*}$ denotes the horizontal convolution, and $\nabla_{h}^{\perp}=\left(-\frac{\partial}{\partial y}, \frac{\partial}{\partial x}\right)$. 
The questions of global well-posedness and the blowup of smooth solutions of the three-dimensional Euler equations has been studied by many authors. The Beale-Kato-Majda criterion [5] states that the quantity

$$
\int_{0}^{T}\|\omega(t)\|_{L^{\infty}} d t
$$

controls the blowup; that is if it is finite then the solution of the Euler equations remains as smooth as the initial data, for initial data $\omega_{0} \in H^{s}$, for $s>1$ in $2 \mathrm{D}$ and $s>3 / 2$ in $3 \mathrm{D}$, on the time interval $[0, T]$, otherwise there is a finite blowup. For initial data $\mathbf{u}_{0} \in H^{s}$, for $s>5 / 2$, the three-dimensional Euler equations posses a unique local in time solution $\mathbf{u}(t ; \mathbf{x})$ in the same space $H^{s}$ (cf. [5], [15]). The same result is valid for initial data $\mathbf{u}_{0} \in C^{1, \alpha}$ for $\alpha \in(0,1]$ [14]. The loss of smoothness of weak solutions for the threedimensional Euler equations with initial data $\mathbf{u}_{0} \in C^{0, \alpha}$, with $\alpha \in(0,1)$, is shown in [3]. In other words, the space $C^{1}$ is the critical space (in velocity) for the short time well-posedness of the three-dimensional Euler equations; that is for initial data more regular than $C^{1}$, one has well-posedness of the three-dimensional Euler equations and for less regular initial data one has ill-posedness. For recent surveys concerning the three-dimensional Euler equations see, for example, [2], [4] and [7]. The situation is different for the two-dimensional Euler equations due to the work of Yudovich [18].

Theorem 2.3. [18] Let $\omega^{0} \in L_{h}^{\infty}\left(\mathbb{T}^{2}\right)$, then system (2.5) has a unique weak solution (i.e. solution in the distribution sense) $\omega \in L^{\infty}\left([0, T] ; L_{h}^{\infty}\left(\mathbb{T}^{2}\right)\right)$ corresponding to the initial data $\omega^{0}$ such that $\|\omega\|_{L^{\infty}\left([0, T] ; L_{h}^{\infty}\left(\mathbb{T}^{2}\right)\right)}=\left\|\omega^{0}\right\|_{L_{h}^{\infty}\left(\mathbb{T}^{2}\right)}$.

Theorem 2.4. [10, 11] Let $\mathbf{u}^{0} \in W_{h}^{s, q}\left(\mathbb{R}^{2}\right)$, with $s>1+\frac{2}{q}, 1<q<\infty$. For any $T>0$, there exists a unique solution $\mathbf{u} \in C\left([0, T] ; W_{h}^{s, q}\left(\mathbb{R}^{2}\right)\right) \cap$ $C^{1}\left([0, T] ; W_{h}^{s-1, q}\left(\mathbb{R}^{2}\right)\right)$ and $p \in C\left([0, T] ; W^{s, q}\left(\mathbb{R}^{2}\right)\right)$ for $(2.4)$ such that

$$
\|\mathbf{u}(t)\|_{W_{h}^{s, q}\left(\mathbb{R}^{2}\right)} \leq K(t),
$$

where $K(t)$ is a real-valued continuous function on $0 \leq t<T$, depending on $s, q$ and $\left\|\mathbf{u}^{0}\right\|_{W_{h}^{s, q}\left(\mathbb{R}^{2}\right)}$.

Finally, we recall the following existence and uniqueness theorems for linear transport equations.

Theorem 2.5. [8] Let $p \in[1, \infty]$ and $u^{0} \in L^{p}\left(\mathbb{R}^{n}\right)$. Assume that

$$
b \in L^{1}\left([0, T] ; L_{l o c}^{1}\left(\mathbb{R}^{n}\right)\right), \quad c \in L^{1}\left([0, T] ; L_{l o c}^{1}\left(\mathbb{R}^{n}\right)\right),
$$




$$
c+\nabla \cdot b \in L^{1}\left([0, T] ; L_{l o c}^{q}\left(\mathbb{R}^{n}\right)\right), \quad b \in L^{1}\left([0, T] ; L_{l o c}^{q}\left(\mathbb{R}^{n}\right)\right),
$$

where $\frac{1}{p}+\frac{1}{q}=1$, and

$$
\begin{aligned}
c+\frac{1}{p} \nabla \cdot b & \in L^{1}\left([0, T] ; L^{\infty}\left(\mathbb{R}^{n}\right)\right), & & \text { if } p>1, \\
c, \nabla \cdot b & \in L^{1}\left([0, T] ; L^{\infty}\left(\mathbb{R}^{n}\right)\right), & & \text { if } p=1 .
\end{aligned}
$$

If $f \in L^{1}\left([0, T] ; L^{p}\left(\mathbb{R}^{n}\right)\right)$, then there exists a unique weak solution $u \in$ $L^{\infty}\left([0, T] ; L^{p}\left(\mathbb{R}^{n}\right)\right)$ of

$$
\frac{\partial u}{\partial t}+(b \cdot \nabla) u+c u=f
$$

corresponding to the initial condition $u^{0}$.

Theorem 2.6. [8] Let $u \in L^{\infty}\left([0, T] ; L^{p}\left(\mathbb{R}^{n}\right)\right)$, where $p \in[1, \infty]$, be a solution of

$$
\frac{\partial u}{\partial t}+(b \cdot \nabla) u+c u=0, \quad u(0 ; x)=0 .
$$

Assume that $c, \nabla \cdot b \in L^{1}\left([0, T] ; L^{\infty}\left(\mathbb{R}^{n}\right)\right), b \in L^{1}\left([0, T] ; W_{l o c}^{1, q}\left(\mathbb{R}^{n}\right)\right)$ where $\frac{1}{p}+\frac{1}{q}=1$ and

$$
\frac{b}{1+|x|} \in L^{1}\left([0, T] ; L^{1}\left(\mathbb{R}^{n}\right)\right)+L^{1}\left([0, T] ; L^{\infty}\left(\mathbb{R}^{n}\right)\right) .
$$

Then $u \equiv 0$.

\section{Global well-posedness of strong solutions}

In this section, we aim to prove the global well-posedness of strong solutions of the inviscid system (1.3), subject to periodic boundary conditions, over any fixed arbitrary time interval $[0, T]$. We give a definition for weak solutions of system (1.3) and prove the global existence and uniqueness of such solutions. Later, we give a definition for strong solutions of system (1.3) and prove the well-posedness of such solutions.

Proposition 3.1 (Apriori Estimates). Assume that $\mathbf{u}_{h} \in C^{1}([0, T]$; $\left.C^{\infty}\left(\mathbb{T}^{2}\right)\right), w \in C^{1}\left([0, T] ; C^{\infty}\left(\mathbb{T}^{2}\right)\right)$ and $\rho \in C^{1}\left([0, T] ; C^{\infty}\left(\mathbb{T}^{3}\right)\right)$ are solutions 
of system (1.3) on the time interval $[0, T]$, subject to periodic boundary conditions. Then, the following estimates hold:

$$
\begin{aligned}
& \sup _{0 \leq t \leq T}\left(\|w(t)\|_{L_{h}^{2}\left(\mathbb{T}^{2}\right)}^{2}+\left\|\langle\rho(t)\rangle_{z}\right\|_{L_{h}^{2}\left(\mathbb{T}^{2}\right)}^{2}\right) \\
& \quad=\left\|w^{0}\right\|_{L_{h}^{2}\left(\mathbb{T}^{2}\right)}^{2}+\left\|\left\langle\rho^{0}\right\rangle_{z}\right\|_{L_{h}^{2}\left(\mathbb{T}^{2}\right)}^{2}, \\
& \sup _{0 \leq t \leq T}\|\rho(t)\|_{L^{2}\left(\mathbb{T}^{3}\right)}^{2} \leq\left\|\rho^{0}\right\|_{L^{2}\left(\mathbb{T}^{3}\right)}+K_{0} T,
\end{aligned}
$$

where $K_{0}$ is a constant that depends on the norms of the initial data. Moreover,

$$
\begin{aligned}
& \sup _{0 \leq t \leq T}\left(\|w(t)\|_{L_{h}^{\infty}\left(\mathbb{T}^{2}\right)}+\left\|\langle\rho(t)\rangle_{z}\right\|_{L_{h}^{\infty}\left(\mathbb{T}^{2}\right)}\right) \\
& \quad \leq\left(\left\|w^{0}\right\|_{L_{h}^{\infty}\left(\mathbb{T}^{2}\right)}+\left\|\left\langle\rho^{0}\right\rangle_{z}\right\|_{L_{h}^{\infty}\left(\mathbb{T}^{2}\right)}\right) e^{T / F r},
\end{aligned}
$$

and

$$
\begin{aligned}
& \sup _{0 \leq t \leq T}\left(\left\|\nabla_{h} w(t)\right\|_{L_{h}^{2}\left(\mathbb{T}^{2}\right)}^{2}+\left\|\nabla_{h}\langle\rho(t)\rangle_{z}\right\|_{L_{h}^{2}\left(\mathbb{T}^{2}\right)}^{2}\right) \\
& \quad \leq\left(\left\|\nabla_{h} w^{0}\right\|_{L_{h}^{2}\left(\mathbb{T}^{2}\right)}^{2}+\left\|\nabla_{h}\left\langle\rho^{0}\right\rangle_{z}\right\|_{L_{h}^{2}\left(\mathbb{T}^{2}\right)}^{2}\right) e^{\int_{0}^{T} 2\left\|\nabla_{h} \mathbf{u}_{h}(s)\right\|_{L_{h}^{\infty}\left(\mathbb{T}^{2}\right)} d s} .
\end{aligned}
$$

Proof. Taking the $L_{h}^{2}\left(\mathbb{T}^{2}\right)$ inner product of (1.3b) with $w$ and (1.3c) with $\langle\rho\rangle_{z}$ yield

$$
\begin{aligned}
\frac{1}{2} \frac{d}{d t}\|w\|_{L_{h}^{2}\left(\mathbb{T}^{2}\right)}^{2} & =-\frac{1}{F r}\left(\langle\rho\rangle_{z}, w\right)_{h}, \\
\frac{1}{2} \frac{d}{d t}\left\|\langle\rho\rangle_{z}\right\|_{L_{h}^{2}\left(\mathbb{T}^{2}\right)}^{2} & =\frac{1}{F r}\left(w,\langle\rho\rangle_{z}\right)_{h} .
\end{aligned}
$$

Adding the above equations implies that

$$
\frac{d}{d t}\left(\|w\|_{L_{h}^{2}\left(\mathbb{T}^{2}\right)}^{2}+\left\|\langle\rho\rangle_{z}\right\|_{L_{h}^{2}\left(\mathbb{T}^{2}\right)}^{2}\right)=0
$$

Integrating the above equation with respect to time on $[0, t]$ proves $(3.1)$.

Taking the $L^{2}\left(\mathbb{T}^{3}\right)$ inner product of $(1.3 \mathrm{~d})$ with $\rho$, and using Young's inequality, yield

$$
\frac{1}{2} \frac{d}{d t}\|\rho\|_{L^{2}\left(\mathbb{T}^{3}\right)}^{2}=\frac{1}{F r}(w, \rho) \leq \frac{1}{F r}\|w\|_{L^{2}\left(\mathbb{T}^{3}\right)}\|\rho\|_{L^{2}\left(\mathbb{T}^{3}\right)}
$$




$$
\begin{aligned}
& =\frac{L^{1 / 2}}{F r}\|w\|_{L_{h}^{2}\left(\mathbb{T}^{2}\right)}\|\rho\|_{L^{2}\left(\mathbb{T}^{3}\right)} \\
& \leq K_{0}\|\rho\|_{L^{2}\left(\mathbb{T}^{3}\right)}
\end{aligned}
$$

where

$$
K_{0}:=\frac{L^{1 / 2}}{F r}\left(\left\|w^{0}\right\|_{L_{h}^{2}\left(\mathbb{T}^{2}\right)}^{2}+\left\|\left\langle\rho^{0}\right\rangle_{z}\right\|_{L_{h}^{2}\left(\mathbb{T}^{2}\right)}^{2}\right)^{1 / 2} .
$$

Thus, we can conclude that

$$
\frac{d}{d t}\|\rho\|_{L^{2}\left(\mathbb{T}^{3}\right)} \leq K_{0}
$$

Integrating the above inequality with respect to time on $[0, t]$, we get that

$$
\|\rho(t)\|_{L^{2}\left(\mathbb{T}^{3}\right)}^{2} \leq\left\|\rho^{0}\right\|_{L^{2}\left(\mathbb{T}^{3}\right)}+K_{0} t
$$

for all $t \in[0, T]$. This proves (3.2).

Now, we multiply $(1.3 \mathrm{~b})$ and $(1.3 \mathrm{c})$ by $w^{2 k-1}$ and $\langle\rho\rangle_{z}^{2 k-1}$, where $k \in \mathbb{N}$, respectively, and integrate over $\mathbb{T}^{2}$. Using Hölder inequality, we have

$$
\begin{aligned}
\frac{1}{2 k} \frac{d}{d t} \int_{\mathbb{T}^{2}} w^{2 k} d \mathbf{x}_{h}=-\frac{1}{F r}\left(\langle\rho\rangle_{z}, w^{2 k-1}\right)_{h} & \leq \frac{1}{F r}\left\|\langle\rho\rangle_{z}\right\|_{L_{h}^{2 k}\left(\mathbb{T}^{2}\right)}\|w\|_{L_{h}^{2 k-1}\left(\mathbb{T}^{2}\right)} \\
& =\frac{1}{F r}\left\|\langle\rho\rangle_{z}\right\|_{L_{h}^{2 k}\left(\mathbb{T}^{2}\right)}\|w\|_{L_{h}^{2 k}\left(\mathbb{T}^{2}\right)}^{2 k-1} \\
\frac{1}{2 k} \frac{d}{d t} \int_{\mathbb{T}^{2}}\langle\rho\rangle_{z}^{2 k} d \mathbf{x}_{h}=-\frac{1}{F r}\left(w,\langle\rho\rangle_{z}^{2 k-1}\right)_{h} & \leq \frac{1}{F r}\|w\|_{L_{h}^{2 k}\left(\mathbb{T}^{2}\right)}\left\|\langle\rho\rangle_{z}\right\|_{L_{h}^{2 k-1}\left(\mathbb{T}^{2}\right)} \\
& =\frac{1}{F r}\|w\|_{L_{h}^{2 k}\left(\mathbb{T}^{2}\right)}\left\|\langle\rho\rangle_{z}\right\|_{L_{h}^{2 k}\left(\mathbb{T}^{2}\right)}^{2 k-1} .
\end{aligned}
$$

Thus,

$$
\begin{aligned}
& \frac{d}{d t}\|w(t)\|_{L_{h}^{2 k}\left(\mathbb{T}^{2}\right)} \leq \frac{1}{F r}\left\|\langle\rho\rangle_{z}\right\|_{L_{h}^{2 k}\left(\mathbb{T}^{2}\right)}, \quad \text { and } \\
& \frac{d}{d t}\left\|\langle\rho\rangle_{z}\right\|_{L_{h}^{2 k}\left(\mathbb{T}^{2}\right)} \leq \frac{1}{F r}\|w\|_{L_{h}^{2 k}\left(\mathbb{T}^{2}\right)} .
\end{aligned}
$$

Adding the above equations and integrating over the time interval $[0, t]$, for $t \leq T$, imply that

$$
\begin{aligned}
& \|w(t)\|_{L_{h}^{2 k}\left(\mathbb{T}^{2}\right)}+\left\|\langle\rho(t)\rangle_{z}\right\|_{L_{h}^{2 k}\left(\mathbb{T}^{2}\right)} \\
& \quad \leq\left(\left\|w^{0}\right\|_{L_{h}^{2 k}\left(\mathbb{T}^{2}\right)}+\left\|\left\langle\rho^{0}\right\rangle_{z}\right\|_{L_{h}^{2 k}\left(\mathbb{T}^{2}\right)}\right) e^{t / F r},
\end{aligned}
$$


for all $t \in[0, T]$ and $k \in \mathbb{N}$. Since the domain is bounded, and the right-hand side bound converges, as $k \rightarrow \infty$, we can take $k \rightarrow \infty$ and obtain

$$
\begin{aligned}
& \left(\|w(t)\|_{L_{h}^{\infty}\left(\mathbb{T}^{2}\right)}+\left\|\langle\rho(t)\rangle_{z}\right\|_{L_{h}^{\infty}\left(\mathbb{T}^{2}\right)}\right) \\
& \quad \leq\left(\left\|w^{0}\right\|_{L_{h}^{\infty}\left(\mathbb{T}^{2}\right)}+\left\|\left\langle\rho^{0}\right\rangle_{z}\right\|_{L_{h}^{\infty}\left(\mathbb{T}^{2}\right)}\right) e^{t / F r},
\end{aligned}
$$

for all $t \in[0, T]$. This proves (3.3).

Taking the $L_{h}^{2}\left(\mathbb{T}^{2}\right)$ inner product of (1.3b) with $-\Delta_{h} w$, and (1.3c) with $-\Delta_{h}\langle\rho\rangle_{z}$, we get that

$\frac{1}{2} \frac{d}{d t}\left\|\nabla_{h} w\right\|_{L_{h}^{2}\left(\mathbb{T}^{2}\right)}^{2} \leq\left\|\nabla_{h} \mathbf{u}_{h}\right\|_{L_{h}^{\infty}\left(\mathbb{T}^{2}\right)}\left\|\nabla_{h} w\right\|_{L_{h}^{2}\left(\mathbb{T}^{2}\right)}^{2}-\frac{1}{F r}\left(\nabla_{h}\langle\rho\rangle_{z}, \nabla_{h} w\right)_{h}$,
$\frac{1}{2} \frac{d}{d t}\left\|\nabla_{h}\langle\rho\rangle_{z}\right\|_{L_{h}^{2}\left(\mathbb{T}^{2}\right)}^{2} \leq\left\|\nabla_{h} \mathbf{u}_{h}\right\|_{L_{h}^{\infty}\left(\mathbb{T}^{2}\right)}\left\|\nabla_{h}\langle\rho\rangle_{z}\right\|_{L_{h}^{2}\left(\mathbb{T}^{2}\right)}^{2}+\frac{1}{F r}\left(\nabla_{h} w, \nabla_{h}\langle\rho\rangle_{z}\right)_{h}$.

Adding the above equations, then integrating with respect to time on $[0, t]$, prove (3.4). This completes the proof.

Proposition 3.2 (Apriori Estimates). Assume that $\mathbf{u}_{h} \in C^{1}([0, T]$; $\left.C^{\infty}\left(\mathbb{T}^{2}\right)\right), w \in C^{1}\left([0, T] ; C^{\infty}\left(\mathbb{T}^{2}\right)\right)$ and $\rho \in C^{1}\left([0, T] ; C^{\infty}\left(\mathbb{T}^{3}\right)\right)$ are solutions of the system (1.3) on the time interval $[0, T]$, subject to periodic boundary conditions. Then, the following estimates hold:

$$
\begin{aligned}
& \sup _{0 \leq t \leq T}\|\rho(t)\|_{L^{\infty}\left(\mathbb{T}^{3}\right)} \\
& \quad \leq\left\|\rho^{0}\right\|_{L^{\infty}\left(\mathbb{T}^{3}\right)}+\left(\left\|w^{0}\right\|_{L_{h}^{\infty}\left(\mathbb{T}^{2}\right)}+\left\|\left\langle\rho^{0}\right\rangle_{z}\right\|_{L_{h}^{\infty}\left(\mathbb{T}^{2}\right)}\right) e^{T / F r}, \\
& \sup _{0 \leq t \leq T}\left(\left\|\nabla_{h} w(t)\right\|_{L_{h}^{\infty}\left(\mathbb{T}^{2}\right)}+\left\|\nabla_{h}\langle\rho(t)\rangle_{z}\right\|_{L_{h}^{\infty}\left(\mathbb{T}^{2}\right)}\right) \\
& \quad \leq \tilde{K}_{0} e^{\int_{0}^{T}\left(1+\left\|\nabla_{h} \mathbf{u}_{h}(s)\right\|_{L_{h}^{\infty}\left(\mathbb{T}^{2}\right)}\right) d s}
\end{aligned}
$$

where $\tilde{K}_{0}$ is a constant that depends on the norms of the initial data.

Proof. We define

$$
\phi(t):=\left\|\rho^{0}\right\|_{L^{\infty}\left(\mathbb{T}^{3}\right)}+\left(\left\|w^{0}\right\|_{L_{h}^{\infty}\left(\mathbb{T}^{2}\right)}+\left\|\left\langle\rho^{0}\right\rangle_{z}\right\|_{L_{h}^{\infty}\left(\mathbb{T}^{2}\right)}\right) e^{t / F r},
$$

and we denote by $\Theta:=\rho-\phi(t)$. Notice that

$$
\frac{\partial \rho}{\partial t}=\frac{\partial \Theta}{\partial t}+\frac{d \phi}{d t}=\frac{\partial \Theta}{\partial t}+\frac{1}{F r}\left(\left\|w^{0}\right\|_{L_{h}^{\infty}\left(\mathbb{T}^{2}\right)}+\left\|\left\langle\rho^{0}\right\rangle_{z}\right\|_{L_{h}^{\infty}\left(\mathbb{T}^{2}\right)}\right) e^{t / F r}
$$




$$
=\frac{\partial \Theta}{\partial t}+\frac{1}{F r}\left(\phi(t)-\left\|\rho^{0}\right\|_{L^{\infty}\left(\mathbb{T}^{3}\right)}\right)
$$

Then, $\Theta$ satisfies the evolution equation:

$$
\frac{\partial \Theta}{\partial t}+\frac{1}{F r}\left(\phi(t)-\left\|\rho^{0}\right\|_{L^{\infty}\left(\mathbb{T}^{3}\right)}\right)+(\mathbf{u} \cdot \nabla) \Theta=\frac{1}{F r} w
$$

We can take the inner product of (3.11) with $\Theta^{+}$and obtain

$$
\begin{aligned}
\frac{1}{2} \frac{d}{d t}\left\|\Theta^{+}(t)\right\|_{L^{2}\left(\mathbb{T}^{3}\right)}^{2}= & \frac{1}{F r}\left(\left(w, \Theta^{+}\right)+\left(\left\|\rho^{0}\right\|_{L^{\infty}\left(\mathbb{T}^{3}\right)}-\phi(t), \Theta^{+}\right)\right) \\
& -\int_{\mathbb{T}^{2}}(\mathbf{u} \cdot \nabla) \Theta \Theta^{+} d \mathbf{x} \\
= & \frac{1}{F r}\left(\left(w, \Theta^{+}\right)+\left(\left\|\rho^{0}\right\|_{L^{\infty}\left(\mathbb{T}^{3}\right)}-\phi(t), \Theta^{+}\right)\right) \\
& -\frac{1}{2} \int_{\mathbb{T}^{2}}(\mathbf{u} \cdot \nabla)\left(\Theta^{+}\right)^{2} d \mathbf{x}, \\
= & \frac{1}{F r}\left(\left(w, \Theta^{+}\right)+\left(\left\|\rho^{0}\right\|_{L^{\infty}\left(\mathbb{T}^{3}\right)}-\phi(t), \Theta^{+}\right)\right),
\end{aligned}
$$

thanks to divergence free condition, $\nabla \cdot \mathbf{u}=0$. Thus,

$$
\begin{aligned}
& \frac{1}{2} \frac{d}{d t}\left\|\Theta^{+}(t)\right\|_{L^{2}\left(\mathbb{T}^{3}\right)}^{2} \\
& \quad \leq \frac{1}{F r}\left(\|w\|_{L^{\infty}\left([0, t] ; L_{h}^{\infty}\left(\mathbb{T}^{2}\right)\right.}+\left\|\rho^{0}\right\|_{L^{\infty}\left(\mathbb{T}^{3}\right)}-\phi(t)\right)\left\|\Theta^{+}\right\|_{L^{1}\left(\mathbb{T}^{3}\right)} .
\end{aligned}
$$

Using (3.8), and the definition of $\phi(t)$, we get that the right-hand side of $(3.12)$ is $\leq 0$. Then,

$$
\frac{d\left\|\Theta^{+}\right\|_{L^{2}\left(\mathbb{T}^{3}\right)}^{2}}{d t} \leq 0, \quad \text { which implies that }\left\|\Theta^{+}(t)\right\|_{L^{2}\left(\mathbb{T}^{3}\right)}^{2} \leq\left\|\Theta^{+}(0)\right\|_{L^{2}\left(\mathbb{T}^{3}\right)}^{2},
$$

for any $t \in[0, T]$. Notice that

$$
\begin{aligned}
\Theta(0 ; \mathbf{x}) & =\rho(0 ; \mathbf{x})-\left\|\rho^{0}\right\|_{L^{\infty}\left(\mathbb{T}^{3}\right)}-\frac{1}{F r}\left(\left\|w^{0}\right\|_{L_{h}^{\infty}\left(\mathbb{T}^{2}\right)}+\left\|\left\langle\rho^{0}\right\rangle_{z}\right\|_{L_{h}^{\infty}\left(\mathbb{T}^{2}\right)}\right) e^{t / F r} \\
& \leq 0
\end{aligned}
$$

Thus, $\Theta^{+}(0, \mathbf{x})=0$ for all $\mathbf{x} \in \mathbb{T}^{3}$. This implies that $\left\|\Theta^{+}(t)\right\|_{L^{\infty}\left(\mathbb{T}^{3}\right)}=0$, for all $t \in[0, T]$. That is, $\Theta^{+}(t ; \mathbf{x})=0$ a.e $\mathbf{x} \in \mathbb{T}^{3}$, for all $t \in[0, T]$. This yield

$$
\rho(t ; \mathbf{x}) \leq\left\|\rho^{0}\right\|_{L^{\infty}\left(\mathbb{T}^{3}\right)}+\frac{1}{F r}\left(\left\|w^{0}\right\|_{L_{h}^{\infty}\left(\mathbb{T}^{2}\right)}+\left\|\left\langle\rho^{0}\right\rangle_{z}\right\|_{L_{h}^{\infty}\left(\mathbb{T}^{2}\right)}\right) e^{t / F r}
$$


for a.e. $\mathbf{x} \in \mathbb{T}^{3}$ and all $t \in[0, T]$. This proves (3.9).

To simplify the notations in the proof, we denote by

$$
\begin{aligned}
R\left(t ; \mathbf{x}_{h}\right) & :=\langle\rho\rangle_{z}\left(t ; \mathbf{x}_{h}\right), \\
Q_{w}^{\lambda}\left(t ; \mathbf{x}_{h}\right) & :=\sqrt{\left|\nabla_{h} w\left(t ; \mathbf{x}_{h}\right)\right|^{2}+\lambda} \quad \text { and } \quad Q_{R}^{\lambda}\left(t ; \mathbf{x}_{h}\right):=\sqrt{\left|\nabla_{h} R\left(t ; \mathbf{x}_{h}\right)\right|^{2}+\lambda},
\end{aligned}
$$

where $\lambda>0$ is any positive number. We choose $\lambda>0$ here so that $Q_{w}^{\lambda}\left(t ; \mathbf{x}_{h}\right)$ and $Q_{R}^{\lambda}\left(t ; \mathbf{x}_{h}\right)$ are strictly positive and real-valued. Taking the $\frac{\partial}{\partial x_{j}}$ of $(1.3 \mathrm{~b})$ and $(1.3 \mathrm{c})$, we obtain the following system

$$
\begin{aligned}
& \frac{\partial}{\partial t} \frac{\partial w}{\partial x_{j}}+\left(\frac{\partial \mathbf{u}_{h}}{\partial x_{j}} \cdot \nabla_{h}\right) w+\left(\mathbf{u}_{h} \cdot \nabla_{h}\right) \frac{\partial w}{\partial x_{j}}=-\frac{1}{F r} \frac{\partial R}{\partial x_{j}}, \\
& \frac{\partial}{\partial t} \frac{\partial R}{\partial x_{j}}+\left(\frac{\partial \mathbf{u}_{h}}{\partial x_{j}} \cdot \nabla_{h}\right) R+\left(\mathbf{u}_{h} \cdot \nabla_{h}\right) \frac{\partial R}{\partial x_{j}}=\frac{1}{F r} \frac{\partial w}{\partial x_{j}},
\end{aligned}
$$

for $j=1,2$. Since

$$
\frac{\partial Q_{w}^{\lambda}}{\partial t}=\frac{\nabla_{h} w}{Q_{w}^{\lambda}} \cdot \frac{\partial \nabla_{h} w}{\partial t}, \quad \text { and } \quad \frac{\partial Q_{R}^{\lambda}}{\partial t}=\frac{\nabla_{h} R}{Q_{R}^{\lambda}} \cdot \frac{\partial \nabla_{h} R}{\partial t},
$$

we take the inner product of (3.14) and (3.15) with $\frac{\frac{\partial w}{\partial x_{j}}}{Q_{w}^{\lambda}}$ and $\frac{\frac{\partial R}{\partial x_{j}}}{Q_{R}^{\lambda}}$, respectively, and then sum over $j=1,2$ and obtain

$$
\begin{aligned}
& \frac{\partial Q_{w}^{\lambda}}{\partial t}+\sum_{j=1}^{2}\left(\frac{\partial \mathbf{u}_{h}}{\partial x_{j}} \cdot \nabla_{h}\right) w \frac{\frac{\partial w}{\partial x_{j}}}{Q_{w}^{\lambda}}+\left(\mathbf{u}_{h} \cdot \nabla_{h}\right) Q_{w}^{\lambda}=-\frac{1}{F r} \nabla_{h} R \frac{\nabla_{h} w}{Q_{w}^{\lambda}} \\
& \frac{\partial Q_{R}^{\lambda}}{\partial t}+\sum_{j=1}^{2}\left(\frac{\partial \mathbf{u}_{h}}{\partial x_{j}} \cdot \nabla_{h}\right) R \frac{\frac{\partial R}{\partial x_{j}}}{Q_{R}^{\lambda}}+\left(\mathbf{u}_{h} \cdot \nabla_{h}\right) Q_{R}^{\lambda}=\frac{1}{F r} \nabla_{h} w \frac{\nabla_{h} R}{Q_{R}^{\lambda}}
\end{aligned}
$$

Now, we define

$$
\phi_{\lambda}(t):=\left(\sqrt{\left\|\nabla_{h} w^{0}\right\|_{L_{h}^{\infty}\left(\mathbb{T}^{2}\right)}+\lambda}+\sqrt{\left\|\nabla_{h}\left\langle\rho^{0}\right\rangle_{z}\right\|_{L_{h}^{\infty}\left(\mathbb{T}^{2}\right)}+\lambda}\right) e^{\int_{0}^{t} \psi(s) d s},
$$

where $\psi(s):=\left\|\nabla_{h} \mathbf{u}_{h}(s)\right\|_{L_{h}^{\infty}\left(\mathbb{T}^{2}\right)}+\frac{1}{F r}$. Denote by $\Theta_{w, \lambda}:=Q_{w}^{\lambda}-\phi_{\lambda}$ and by $\Theta_{R, \lambda}:=Q_{R}^{\lambda}-\phi_{\lambda}$. It is clear that $\frac{\partial \phi_{\lambda}}{\partial t}(t)=\psi(t) \phi_{\lambda}(t)$, thus we can replace $\frac{\partial Q_{w}^{\lambda}}{\partial t}$ in (3.16) by $\frac{\partial \Theta_{w, \lambda}}{\partial t}+\psi \phi_{\lambda}$. Then, we take the inner product of the equation 
with $\Theta_{w, \lambda}^{+}$and obtain

$$
\frac{1}{2} \frac{d}{d t}\left\|\Theta_{w, \lambda}^{+}\right\|_{L_{h}^{2}\left(\mathbb{T}^{2}\right)}^{2}+J_{1}+J_{2}=J_{3}+J_{4}
$$

where

$$
\begin{array}{ll}
J_{1}:=\sum_{j=1}^{2} \int_{\mathbb{T}^{2}}\left(\frac{\partial \mathbf{u}_{h}}{\partial x_{j}} \cdot \nabla_{h}\right) w \frac{\partial w}{\partial x_{j}} \Theta_{w}^{\lambda} \Theta_{w, \lambda} d \mathbf{x}_{h}, & J_{2}:=\int_{\mathbb{T}^{2}}\left(\mathbf{u}_{h} \cdot \nabla_{h}\right) \Theta_{w, \lambda}^{+} \Theta_{w, \lambda}^{+} d \mathbf{x}_{h}, \\
J_{3}:=-\frac{1}{F r} \int_{\mathbb{T}^{2}} \frac{\nabla_{h} R \cdot \nabla_{h} w}{Q_{w}^{\nu, \lambda}} \Theta_{w, \lambda}^{+} d \mathbf{x}_{h}, & J_{4}:=-\phi_{\lambda} \psi \int_{\mathbb{T}^{2}} \Theta_{w, \lambda}^{+} d \mathbf{x}_{h} .
\end{array}
$$

The divergence free condition, $\nabla_{h} \cdot \mathbf{u}_{h}=0$, implies that $J_{2}=0$. By CauchySchwarz inequality,

$$
\begin{aligned}
\left|J_{1}\right| \leq & \int_{\mathbb{T}^{2}} \frac{\left|\nabla_{h} \mathbf{u}_{h}\right|\left|\nabla_{h} w\right|^{2}}{Q_{w}^{\lambda}} \Theta_{w, \lambda}^{+} d \mathbf{x}_{h} \\
\leq & \left\|\nabla_{h} \mathbf{u}_{h}\right\|_{L_{h}^{\infty}\left(\mathbb{T}^{2}\right)} \int_{\mathbb{T}^{2}}\left(\Theta_{w, \lambda}+\phi_{\lambda}\right) \Theta_{w, \lambda}^{+} d \mathbf{x}_{h} \\
= & \left\|\nabla_{h} \mathbf{u}_{h}\right\|_{L_{h}^{\infty}\left(\mathbb{T}^{2}\right)} \int_{\mathbb{T}^{2}}\left(\Theta_{w, \lambda}^{+}\right)^{2} d \mathbf{x}_{h} \\
& +\left\|\nabla_{h} \mathbf{u}_{h}\right\|_{L_{h}^{\infty}\left(\mathbb{T}^{2}\right)} \phi_{\lambda} \int_{\mathbb{T}^{2}} \Theta_{w, \lambda}^{+} d \mathbf{x}_{h},
\end{aligned}
$$

and

$$
\begin{aligned}
\left|J_{3}\right| \leq & \frac{1}{F r} \int_{\mathbb{T}^{2}} \frac{\left|\nabla_{h} R\right|\left|\nabla_{h} w\right|}{Q_{w}^{\lambda}} \Theta_{w, \lambda}^{+} d \mathbf{x}_{h} \\
\leq & \frac{1}{F r} \int_{\mathbb{T}^{2}} Q_{R}^{\lambda} \Theta_{w, \lambda}^{+} d \mathbf{x}_{h} \\
\leq & \frac{1}{F r} \int_{\mathbb{T}^{2}} \Theta_{R, \lambda} \Theta_{w, \lambda}^{+} d \mathbf{x}_{h}+\frac{1}{F r} \phi_{\lambda} \int_{\mathbb{T}^{2}} \Theta_{w, \lambda}^{+} d \mathbf{x}_{h} \\
\leq & \frac{1}{F r} \int_{\mathbb{T}^{2}} \Theta_{R, \lambda}^{+} \Theta_{w, \lambda}^{+} d \mathbf{x}_{h}+\frac{1}{F r} \phi_{\lambda} \int_{\mathbb{T}^{2}} \Theta_{w, \lambda}^{+} d \mathbf{x}_{h} \\
= & \frac{1}{2 F r} \int_{\mathbb{T}^{2}}\left(\Theta_{R, \lambda}^{+}\right)^{2} d \mathbf{x}_{h}+\frac{1}{2 F r} \int_{\mathbb{T}^{2}}\left(\Theta_{w, \lambda}^{+}\right)^{2} d \mathbf{x}_{h} \\
& +\frac{1}{F r} \phi_{\lambda} \int_{\mathbb{T}^{2}} \Theta_{w, \lambda}^{+} d \mathbf{x}_{h},
\end{aligned}
$$


where we used Young's inequality in the last step. Finally, from (3.18) and (3.19) we have

$$
\begin{aligned}
\frac{1}{2} \frac{d}{d t}\left\|\Theta_{w, \lambda}^{+}\right\|_{L_{h}^{2}\left(\mathbb{T}^{2}\right)}^{2} \leq & \left\|\nabla_{h} \mathbf{u}_{h}\right\|_{L_{h}^{\infty}\left(\mathbb{T}^{2}\right)}\left\|\Theta_{w, \lambda}^{+}\right\|_{L_{h}^{2}\left(\mathbb{T}^{2}\right)}^{2} \\
& +\left\|\nabla_{h} \mathbf{u}_{h}\right\|_{L_{h}^{\infty}\left(\mathbb{T}^{2}\right)} \phi_{\lambda}\left\|\Theta_{w, \lambda}^{+}\right\|_{L_{h}^{1}\left(\mathbb{T}^{2}\right)} \\
& +\frac{1}{2 F r}\left\|\Theta_{w, \lambda}^{+}\right\|_{L_{h}^{2}\left(\mathbb{T}^{2}\right)}^{2}+\frac{1}{2 F r}\left\|\Theta_{R, \lambda}^{+}\right\|_{L_{h}^{2}\left(\mathbb{T}^{2}\right)}^{2} \\
& +\frac{1}{F r} \phi_{\lambda}\left\|\Theta_{w, \lambda}^{+}\right\|_{L_{h}^{1}\left(\mathbb{T}^{2}\right)}-\phi_{\lambda} \psi\left\|\Theta_{w, \lambda}^{+}\right\|_{L_{h}^{1}\left(\mathbb{T}^{2}\right)} .
\end{aligned}
$$

Similar argument will yield a similar inequality for $\Theta_{R, \lambda}^{+}$. After summing the two inequalities we get

$$
\begin{aligned}
& \frac{1}{2} \frac{d}{d t}\left(\left\|\Theta_{w, \lambda}^{+}\right\|_{L_{h}^{2}\left(\mathbb{T}^{2}\right)}^{2}+\left\|\Theta_{R, \lambda}^{+}\right\|_{L_{h}^{2}\left(\mathbb{T}^{2}\right)}^{2}\right) \leq \\
& \quad\left(\left\|\nabla_{h} \mathbf{u}_{h}\right\|_{L_{h}^{\infty}\left(\mathbb{T}^{2}\right)}+\frac{1}{F r}\right)\left(\left\|\Theta_{w, \lambda}^{+}\right\|_{L_{h}^{2}\left(\mathbb{T}^{2}\right)}^{2}+\left\|\Theta_{R, \lambda}^{+}\right\|_{L_{h}^{2}\left(\mathbb{T}^{2}\right)}^{2}\right) \\
& \quad+\phi_{\lambda}\left(\left\|\Theta_{w, \lambda}^{+}\right\|_{L_{h}^{1}\left(\mathbb{T}^{2}\right)}+\left\|\Theta_{R, \lambda}^{+}\right\|_{L_{h}^{1}\left(\mathbb{T}^{2}\right)}\right)\left(\left\|\nabla_{h} \mathbf{u}_{h}\right\|_{L_{h}^{\infty}\left(\mathbb{T}^{2}\right)}+\frac{1}{F r}-\psi\right) .
\end{aligned}
$$

Since $\left\|\nabla_{h} \mathbf{u}_{h}\right\|_{L_{h}^{\infty}\left(\mathbb{T}^{2}\right)}+\frac{1}{F r}-\psi=0$, by the definition of $\psi$, then integrating with respect to time over the interval $[0, t]$, for $t \leq T$, yields

$$
\begin{aligned}
\| \Theta_{w, \lambda}^{+}(t) & \left\|_{L_{h}^{2}\left(\mathbb{T}^{2}\right)}^{2}+\right\| \Theta_{R, \lambda}^{+}(t) \|_{L_{h}^{2}\left(\mathbb{T}^{2}\right)}^{2} \\
& \leq\left(\left\|\Theta_{w, \lambda}^{+}(0)\right\|_{L_{h}^{2}\left(\mathbb{T}^{2}\right)}^{2}+\left\|\Theta_{R, \lambda}^{+}(0)\right\|_{L_{h}^{2}\left(\mathbb{T}^{2}\right)}^{2}\right) e^{\int_{0}^{t} 2 \psi(s) d s} .
\end{aligned}
$$

Since $\Theta_{w, \lambda}(0)=\sqrt{\left|\nabla_{h} w^{0}\right|^{2}+\lambda}-\phi_{\lambda}(0) \leq 0$, then $\Theta_{w, \lambda}^{+}(0)=0$. By a similar argument, $\Theta_{R, \lambda}^{+}(0)=0$. Therefore, for all $t \in[0, T]$ and a.e $\mathbf{x}_{h} \in \mathbb{T}^{2}$,

$$
\begin{aligned}
\sqrt{\left|\nabla_{h} w(t)\right|^{2}+\lambda} & \leq \phi_{\lambda}(t) \\
& =\left(\sqrt{\left\|\nabla_{h} w^{0}\right\|_{L_{h}^{\infty}\left(\mathbb{T}^{2}\right)}^{2}+\lambda}+\sqrt{\left\|\nabla_{h} R^{0}\right\|_{L_{h}^{\infty}\left(\mathbb{T}^{2}\right)}^{2}+\lambda}\right) e^{\int_{0}^{t} \psi(s) d s},
\end{aligned}
$$




$$
\begin{aligned}
\sqrt{\left|\nabla_{h} R(t)\right|^{2}+\lambda} & \leq \phi_{\lambda}(t) \\
& =\left(\sqrt{\left\|\nabla_{h} w^{0}\right\|_{L_{h}^{\infty}\left(\mathbb{T}^{2}\right)}^{2}+\lambda}+\sqrt{\left\|\nabla_{h} R^{0}\right\|_{L_{h}^{\infty}\left(\mathbb{T}^{2}\right)}^{2}+\lambda}\right) e^{\int_{0}^{t} \psi(s) d s} .
\end{aligned}
$$

This implies that

$$
\begin{aligned}
& \sqrt{\left\|\nabla_{h} w\right\|_{L_{h}^{\infty}\left(\mathbb{T}^{2}\right)}^{2}+\lambda}+\sqrt{\left\|\nabla_{h} R\right\|_{L_{h}^{\infty}\left(\mathbb{T}^{2}\right)}^{2}+\lambda} \leq \\
& \quad 2\left(\sqrt{\left\|\nabla_{h} w^{0}\right\|_{L_{h}^{\infty}\left(\mathbb{T}^{2}\right)}^{2}+\lambda}+\sqrt{\left\|\nabla_{h} R^{0}\right\|_{L_{h}^{\infty}\left(\mathbb{T}^{2}\right)}^{2}+\lambda}\right) e^{\int_{0}^{t} \psi(s) d s},
\end{aligned}
$$

for all $\lambda>0$. Since $\lambda>0$ is arbitrary, then we can take $\lambda \rightarrow 0^{+}$in the above inequality. This proves (3.10) and completes the proof.

Definition 3.3 (Weak Solutions). Let $s>2, \mathbf{u}_{h}^{0}\left(\mathbf{x}_{h}\right) \in H_{h}^{s}\left(\mathbb{T}^{2}\right), w^{0}\left(\mathbf{x}_{h}\right) \in$ $H_{h}^{1}\left(\mathbb{T}^{2}\right) \cap L_{h}^{\infty}\left(\mathbb{T}^{2}\right),\left\langle\rho^{0}\right\rangle_{z}\left(\mathbf{x}_{h}\right) \in H_{h}^{1}\left(\mathbb{T}^{2}\right) \cap L_{h}^{\infty}\left(\mathbb{T}^{2}\right)$ and $\rho^{0} \in L^{2}\left(\mathbb{T}^{3}\right)$. For any $T>0$, we say that $\mathbf{u}_{h}\left(t ; \mathbf{x}_{h}\right), p\left(t ; \mathbf{x}_{h}\right), w\left(t ; \mathbf{x}_{h}\right)$ and $\rho(t ; \mathbf{x})$ is a weak solution of system (1.3), on the time interval $[0, T]$, if

$$
\begin{array}{r}
\mathbf{u}_{h} \in C\left([0, T] ; H_{h}^{s}\left(\mathbb{T}^{2}\right)\right) \cap C^{1}\left([0, T] ; H_{h}^{s-1}\left(\mathbb{T}^{2}\right)\right), \\
p \in C\left([0, T] ; H_{h}^{s}\left(\mathbb{T}^{2}\right)\right), \\
w \in L^{\infty}\left([0, T] ; H_{h}^{1}\left(\mathbb{T}^{2}\right) \cap L_{h}^{\infty}\left(\mathbb{T}^{2}\right)\right), \\
\langle\rho\rangle_{z} \in L^{\infty}\left([0, T] ; H_{h}^{1}\left(\mathbb{T}^{2}\right) \cap L_{h}^{\infty}\left(\mathbb{T}^{2}\right)\right), \\
\rho \in L^{\infty}\left([0, T] ; L^{2}\left(\mathbb{T}^{3}\right)\right) .
\end{array}
$$

Moreover, $\mathbf{u}_{h}, w$ and $\rho$ satisfy (1.3) in the distribution sense; that is, for any $\phi\left(t ; \mathbf{x}_{h}\right), \chi_{1}\left(t ; \mathbf{x}_{h}\right), \chi_{2}\left(t ; \mathbf{x}_{h}\right) \in \mathcal{D}\left([0, T] \times \mathbb{T}^{2}\right)$, with $\phi\left(T, \mathbf{x}_{h}\right)=\chi_{1}\left(T, \mathbf{x}_{h}\right)=$ $\chi_{2}\left(T, \mathbf{x}_{h}\right)=0$, and any $\psi(t ; \mathbf{x}) \in \mathcal{D}\left([0, T] \times \mathbb{T}^{3}\right)$ with $\psi(T, \mathbf{x})=0$, such that $\nabla_{h} \cdot \phi=\nabla_{h} \cdot \chi_{1}=\nabla_{h} \cdot \chi_{2}=\nabla \cdot \psi=0$, the following integral identities hold:

$$
\begin{aligned}
& \int_{0}^{T}\left(\mathbf{u}_{h}(s), \phi^{\prime}(s)\right)_{h} d s+\int_{0}^{T}\left(\left(\mathbf{u}_{h}(s) \cdot \nabla_{h}\right) \phi(s), \mathbf{u}_{h}(s)\right)_{h} d s \\
& \quad=-\left(\mathbf{u}_{h}^{0}, \phi^{0}\right)_{h}, \\
& \int_{0}^{T}\left(w(s), \chi_{1}^{\prime}(s)\right)_{h} d s+\int_{0}^{T}\left(\left(\mathbf{u}_{h}(s) \cdot \nabla_{h}\right) \chi_{1}(s), w(s)\right)_{h} d s \\
& \quad=-\left(w^{0}, \chi_{1}^{0}\right)_{h}+\frac{1}{F r} \int_{0}^{T}\left(\langle\rho(s)\rangle_{z}, \chi_{1}(s)\right)_{h} d s
\end{aligned}
$$




$$
\begin{aligned}
& \int_{0}^{T}\left(\langle\rho\rangle_{z}(s), \chi_{2}^{\prime}(s)\right)_{h} d s+\int_{0}^{T}\left(\left(\mathbf{u}_{h}(s) \cdot \nabla_{h}\right) \chi_{2}(s),\langle\rho\rangle_{z}(s)\right)_{h} d s \\
& =-\left(\left\langle\rho^{0}\right\rangle_{z}, \chi_{2}^{0}\right)_{h}-\frac{1}{F r} \int_{0}^{T}\left(w(s), \chi_{2}(s)\right)_{h} d s, \\
& \int_{0}^{T}\left(\rho(s), \psi^{\prime}(s)\right) d s+\int_{0}^{T}((\mathbf{u}(s) \cdot \nabla) \psi(s), \rho(s)) d s \\
& =-\left(\rho^{0}, \psi^{0}\right)-\frac{1}{F r} \int_{0}^{T}(w(s), \psi(s)) d s .
\end{aligned}
$$

Theorem 3.4 (Global existence and uniqueness of weak solutions). Let $s>2, \mathbf{u}_{h}^{0} \in H_{h}^{s}\left(\mathbb{T}^{2}\right), w^{0} \in H_{h}^{1}\left(\mathbb{T}^{2}\right) \cap L_{h}^{\infty}\left(\mathbb{T}^{2}\right),\left\langle\rho^{0}\right\rangle_{z} \in H_{h}^{1}\left(\mathbb{T}^{2}\right) \cap L_{h}^{\infty}\left(\mathbb{T}^{2}\right)$ and $\rho^{0} \in L^{2}\left(\mathbb{T}^{3}\right)$. Then, for any given $T>0$, system (1.3) has a unique weak solution in the sense of Definition 3.3. Moreover, the solution satisfies the estimates in Proposition 3.1.

Proof. By Theorem 2.4, there exists a unique solution $\mathbf{u}_{h} \in C\left([0, T] ; H_{h}^{s}\left(\mathbb{T}^{2}\right)\right)$ $\cap C^{1}\left([0, T] ; H_{h}^{s-1}\left(\mathbb{T}^{2}\right)\right)$ and $p \in C\left([0, T] ; H_{h}^{s}\left(\mathbb{T}^{2}\right)\right)$ of the incompressible $\left(\nabla_{h}\right.$. $\mathbf{u}_{h}=0$ ) two-dimensional Euler equations (1.3a). The solution is classical and will satisfy (3.25a) and the estimate (2.7). It is clear that $\nabla_{h} \mathbf{u}_{h}$ $\in L^{\infty}\left([0, T] ; L_{h}^{\infty}\left(\mathbb{T}^{2}\right)\right)$, and by the elliptic regularity estimate $(2.1), \mathbf{u}_{h} \in$ $L^{\infty}\left([0, T] ; W_{h}^{1, q}\left(\mathbb{T}^{2}\right)\right) \cap L^{\infty}\left([0, T] ; L_{h}^{\infty}\left(\mathbb{T}^{2}\right)\right)$, for any $q \in[2, \infty)$.

Now we consider the system:

$$
\begin{array}{r}
\frac{\partial w}{\partial t}+\left(\mathbf{u}_{h} \cdot \nabla_{h}\right) w=-\frac{1}{F r}\langle\rho\rangle_{z}, \\
\frac{\partial\langle\rho\rangle_{z}}{\partial t}+\left(\mathbf{u}_{h} \cdot \nabla_{h}\right)\langle\rho\rangle_{z}=\frac{1}{F r} w, \\
w\left(0 ; \mathbf{x}_{h}\right)=w^{0}\left(\mathbf{x}_{h}\right), \quad\langle\rho\rangle_{z}\left(0 ; \mathbf{x}_{h}\right)=\langle\rho\rangle_{z}\left(\mathbf{x}_{h}\right) .
\end{array}
$$

To prove the existence of $w\left(t ; \mathbf{x}_{h}\right)$ and $\langle\rho\rangle_{z}\left(t ; \mathbf{x}_{h}\right)$, we will follow some ideas introduced by DiPerna and Lions in [8]. Let $\eta\left(\mathbf{x}_{h}\right) \in \mathcal{D}\left(\mathbb{R}^{2}\right)$ with $\int_{\mathbb{R}^{2}} \eta\left(\mathbf{x}_{h}\right) d \mathbf{x}_{h}=1$. Consider $\mathbf{u}_{h, \varepsilon}=\mathbf{u}_{h}{ }_{h}^{*} \eta_{\varepsilon}, w_{\varepsilon}^{0}=w_{h}^{0}{ }_{h}^{*} \eta_{\varepsilon},\left\langle\rho_{\varepsilon}^{0}\right\rangle_{z}=\left\langle\rho^{0}\right\rangle_{z}{ }_{h}^{*}$ $\eta_{\varepsilon}$ where $\eta_{\varepsilon}()=.\frac{1}{\varepsilon} \eta(\dot{\bar{\varepsilon}})$. Then, by standard consideration, there exists a unique classical solution $w_{\varepsilon},\left\langle\rho_{\varepsilon}\right\rangle_{z} \in C\left([0, T] ; C_{b}^{1}\left(\mathbb{T}^{2}\right)\right)$ of

$$
\begin{gathered}
\frac{\partial w_{\varepsilon}}{\partial t}+\left(\mathbf{u}_{h, \varepsilon} \cdot \nabla_{h}\right) w_{\varepsilon}=-\frac{1}{F r}\left\langle\rho_{\varepsilon}\right\rangle_{z} \\
\frac{\partial\left\langle\rho_{\varepsilon}\right\rangle_{z}}{\partial t}+\left(\mathbf{u}_{h, \varepsilon} \cdot \nabla_{h}\right)\left\langle\rho_{\varepsilon}\right\rangle_{z}=\frac{1}{F r} w_{\varepsilon}
\end{gathered}
$$




$$
w_{\varepsilon}\left(0 ; \mathbf{x}_{h}\right)=w_{\varepsilon}^{0}\left(\mathbf{x}_{h}\right), \quad\left\langle\rho_{\varepsilon}\right\rangle_{z}\left(0 ; \mathbf{x}_{h}\right)=\left\langle\rho_{\varepsilon}^{0}\right\rangle_{z}\left(\mathbf{x}_{h}\right),
$$

which clearly satisfies $(3.25 \mathrm{~b})$ and $(3.25 \mathrm{c})$. Moreover, the solution $w_{\varepsilon}$ and $\left\langle\rho_{\varepsilon}\right\rangle_{z}$ satisfies the estimates (3.3) and (3.4), for any $\varepsilon>0$. By the BanachAlaoglu compactness theorem, we can extract a subsequence (which we will still denote $\left.\left\{w_{\varepsilon}\right\}_{\varepsilon>0},\left\{\left\langle\rho_{\varepsilon}\right\rangle_{z}\right\}_{\varepsilon>0}\right)$ such that

$$
\begin{aligned}
w_{\varepsilon} \underset{*}{\rightarrow} w, & \text { in } L^{\infty}\left([0, T] ; L_{h}^{\infty}\left(\mathbb{T}^{2}\right)\right), \\
\left\langle\rho_{\varepsilon}\right\rangle_{z} \rightarrow\langle\rho\rangle_{z}, & \text { in } L^{\infty}\left([0, T] ; L_{h}^{\infty}\left(\mathbb{T}^{2}\right)\right),
\end{aligned}
$$

for some $w,\langle\rho\rangle_{z} \in L^{\infty}\left([0, T] ; L_{h}^{\infty}\left(\mathbb{T}^{2}\right)\right)$, as $\varepsilon \rightarrow 0$. The limit $w$ and $\langle\rho\rangle_{z}$ will inherit estimate (3.3). Recall that

$$
\mathbf{u}_{h, \varepsilon} \rightarrow \mathbf{u}_{h}, \quad \text { in } L^{\infty}\left([0, T] ; L_{h}^{1}\left(\mathbb{T}^{2}\right)\right),
$$

as $\varepsilon \rightarrow 0$. The strong convergence (3.29) and the weak-* convergence (3.28) are enough to pass to the limit in (3.25b) and (3.25c) and show that $w$ and $\langle\rho\rangle_{z}$ is a weak solution of system (3.26).

The uniqueness of $w$ and $\langle\rho\rangle_{z}$ will follow by a similar argument as in the proof of Theorem 2.6 of DiPerna and Lions, since $\mathbf{u}_{h} \in L^{\infty}\left([0, T] ; W_{h}^{1, q}\left(\mathbb{T}^{2}\right) \cap\right.$ $\left.L_{h}^{\infty}\left(\mathbb{T}^{2}\right)\right)$, for any $q \in[2, \infty)$. Since the solution $w$ and $\langle\rho\rangle_{z}$ is unique, using the Banach-Alaoglu compactness theorem, we can extract a subsequence (which we will still denote $\left\{w_{\varepsilon}\right\}_{\varepsilon>0},\left\{\left\langle\rho_{\varepsilon}\right\rangle_{z}\right\}_{\varepsilon>0}$ ) such that

$$
\begin{aligned}
\nabla_{h} w_{\varepsilon} \underset{*}{\rightarrow} \nabla_{h} w, & \text { in } L^{\infty}\left([0, T] ; L_{h}^{\infty}\left(\mathbb{T}^{2}\right)\right), \\
\nabla_{h}\left\langle\rho_{\varepsilon}\right\rangle_{z} \underset{*}{\rightarrow} \nabla_{h}\langle\rho\rangle_{z}, & \text { in } L^{\infty}\left([0, T] ; L_{h}^{\infty}\left(\mathbb{T}^{2}\right)\right),
\end{aligned}
$$

as $\varepsilon \rightarrow 0$. Thus, the solution $w$ and $\langle\rho\rangle_{z}$ will inherit estimate (3.4).

The existence and the uniqueness of a solution $\rho(t ; \mathbf{x}) \in L^{\infty}([0, T]$; $\left.L^{2}\left(\mathbb{T}^{3}\right)\right)$ of the linear equation $(1.3 \mathrm{~d})$, that satisfies $(3.25 \mathrm{~d})$, follows by Theorem 2.5 and Theorem 2.6, since $\mathbf{u}_{h}, w \in L^{\infty}\left([0, T] ; H_{h}^{1}\left(\mathbb{T}^{2}\right) \cap L_{h}^{\infty}\left(\mathbb{T}^{2}\right)\right)$. Finally, we recall that the proof of Theorem 2.5 is based on the same idea of constructing an approximate sequence of solutions we used in the above proof. Thus, by using the Banach-Alaoglu compactness theorem, the solution $\rho(t ; \mathbf{x})$ will inherit estimate (3.2). For more details, see the proof of Theorem 2.5 in [8].

Definition 3.5 (Strong Solutions). Let $s>2, \mathbf{u}_{h}^{0}\left(\mathbf{x}_{h}\right) \in H_{h}^{s}\left(\mathbb{T}^{2}\right), w^{0}\left(\mathbf{x}_{h}\right) \in$ $W_{h}^{1, \infty}\left(\mathbb{T}^{2}\right),\left\langle\rho^{0}\right\rangle_{z}\left(\mathbf{x}_{h}\right) \in W_{h}^{1, \infty}\left(\mathbb{T}^{2}\right)$ and $\rho^{0}(\mathbf{x}) \in L^{\infty}\left(\mathbb{T}^{3}\right)$. We say that $\mathbf{u}_{h}\left(t ; \mathbf{x}_{h}\right), p\left(t ; \mathbf{x}_{h}\right), w\left(t ; \mathbf{x}_{h}\right)$ and $\rho(t ; \mathbf{x})$ is a strong solution of system (1.3), on the time interval $[0, T]$, if it is a weak solution of $(1.3)$, in the sense of 
Definition 3.3, and satisfies

$$
\begin{aligned}
w & \in L^{\infty}\left([0, T] ; W_{h}^{1, \infty}\left(\mathbb{T}^{2}\right)\right), \\
\langle\rho\rangle_{z} & \in L^{\infty}\left([0, T] ; W_{h}^{1, \infty}\left(\mathbb{T}^{2}\right)\right), \\
& \rho \in L^{\infty}\left([0, T] ; L^{\infty}\left(\mathbb{T}^{3}\right)\right) .
\end{aligned}
$$

Theorem 3.6 (Global well-posedness of strong solutions). Let $s>2, \mathbf{u}_{h}^{0} \in$ $H_{h}^{s}\left(\mathbb{T}^{2}\right), w^{0} \in W_{h}^{1, \infty}\left(\mathbb{T}^{2}\right),\left\langle\rho^{0}\right\rangle_{z} \in W_{h}^{1, \infty}\left(\mathbb{T}^{2}\right)$ and $\rho^{0} \in L^{\infty}\left(\mathbb{T}^{3}\right)$. Then, for any given $T>0$, system (1.3) has a unique strong solution in the sense of Definition 3.5 that satisfies the estimates in Proposition 3.1 and Proposition 3.2.

Assume that $\mathbf{u}_{h}^{1}, p^{1}, w^{1}, \rho^{1}$ and $\mathbf{u}_{h}^{2}, p^{2}, w^{2}, \rho^{2}$ are two strong solutions of system (1.3), in the sense of Definition 3.5, with corresponding initial data $\mathbf{u}_{h}^{1,0}, w^{1,0}, \rho^{1,0}, \mathbf{u}_{h}^{2,0}, w^{2,0}$ and $\rho^{2,0}$, respectively. Define $\xi^{i}:=(-\Delta)^{-1} \rho^{i}$ and $\xi^{i, 0}:=(-\Delta)^{-1} \rho^{i, 0}$, for $i=1,2$. Then,

$$
\begin{aligned}
D(t) \leq & D(0) e^{C_{0}^{1,2} t} ; \\
D(t):= & \left\|\left(\mathbf{u}_{h}^{1}-\mathbf{u}_{h}^{2}\right)(t)\right\|_{L_{h}^{2}\left(\mathbb{T}^{2}\right)}^{2}+\left\|\left(w^{1}-w^{2}\right)(t)\right\|_{L_{h}^{2}\left(\mathbb{T}^{2}\right)}^{2} \\
& +\left\|\left\langle\rho^{1}-\rho^{2}\right\rangle_{z}(t)\right\|_{L_{h}^{2}\left(\mathbb{T}^{2}\right)}^{2}+\left\|\nabla\left(\xi^{1}-\xi^{2}\right)(t)\right\|_{L^{2}\left(\mathbb{T}^{3}\right)}^{2},
\end{aligned}
$$

for all $t \in[0, T]$, where $C_{0}^{1,2}=C_{0}^{1,2}(L, T, F r)$ is a constant that depends on $T, L, F r$ and may depend on the norms of the initial data $\mathbf{u}_{h}^{1,0}, \mathbf{u}_{h}^{2,0}, w^{1,0}$, $w^{2,0}, \rho^{1,0}$ and $\rho^{2,0}$.

Proof. The existence and uniqueness of a weak solution follows by Theorem 3.4. The solution will satisfy the estimates in Proposition 3.2 by the same argument presented in the proof of Theorem 3.4. This proves that the solution is a strong solution. Assume that $\mathbf{u}_{h}^{1}, p^{1}, w^{1}, \rho^{1}$ and $\mathbf{u}_{h}^{2}, p^{2}, w^{2}$, $\rho^{2}$ are two strong solutions of system (1.3) with corresponding initial data $\mathbf{u}_{h}^{1,0}, w^{1,0}, \rho^{1,0}, \mathbf{u}_{h}^{2,0}, w^{2,0}$ and $\rho^{2,0}$, respectively. Following the idea introduced in [13], we introduce the stream function $\xi^{i}$, where $\rho^{i}:=-\Delta \xi^{i}$ and $\int_{\mathbb{T}^{3}} \xi^{i}(t ; \mathbf{x}) d \mathbf{x}=0$, for all $t \in[0, T]$ with corresponding initial condition $\xi^{i, 0}$, for $i=1,2$. We denote by $\tilde{\mathbf{u}}_{h}:=\mathbf{u}_{h}^{1}-\mathbf{u}_{h}^{2}, \tilde{p}:=p^{1}-p^{2}, \tilde{w}:=w^{1}-w^{2}$, $\tilde{\rho}:=\rho^{1}-\rho^{2}$ and $\tilde{\xi}:=\xi^{1}-\xi^{2}$. It is easy to check that $\tilde{\mathbf{u}}_{h}, \tilde{w}, \tilde{\rho}$ and $\tilde{\xi}$ will satisfy the functional equations

$$
\begin{aligned}
& \frac{\partial \tilde{\mathbf{u}}_{h}}{\partial t}+\left(\mathbf{u}_{h}^{1} \cdot \nabla_{h}\right) \tilde{\mathbf{u}}_{h}+\left(\tilde{\mathbf{u}}_{h} \cdot \nabla_{h}\right) \mathbf{u}_{h}^{2}+\nabla_{h} \tilde{p}=0, \\
& \quad \text { in } C\left([0, T] ; H_{h}^{s-1}\left(\mathbb{T}^{2}\right)\right),
\end{aligned}
$$




$$
\begin{aligned}
& \frac{\partial \tilde{w}}{\partial t}+\left(\mathbf{u}_{h}^{1} \cdot \nabla_{h}\right) \tilde{w}+\left(\tilde{\mathbf{u}}_{h} \cdot \nabla_{h}\right) w^{2}=-\frac{1}{F r}\langle\tilde{\rho}\rangle_{z}, \\
& \quad \operatorname{in~} L^{2}\left([0, T] ; L_{h}^{2}\left(\mathbb{T}^{2}\right)\right), \\
& \frac{\partial\langle\tilde{\rho}\rangle_{z}}{\partial t}+\left(\mathbf{u}_{h}^{1} \cdot \nabla_{h}\right)\langle\tilde{\rho}\rangle_{z}+\left(\tilde{\mathbf{u}}_{h} \cdot \nabla_{h}\right)\left\langle\rho^{2}\right\rangle_{z}=\frac{1}{F r} \tilde{w}, \\
& \quad \operatorname{in~} L^{2}\left([0, T] ; L_{h}^{2}\left(\mathbb{T}^{2}\right)\right), \\
& -\frac{\partial \Delta \tilde{\xi}}{\partial t}-\left(\mathbf{u}_{h}^{1} \cdot \nabla_{h}\right) \Delta \tilde{\xi}-\left(\tilde{\mathbf{u}}_{h} \cdot \nabla_{h}\right) \Delta \xi^{2}-w^{1} \frac{\partial \Delta \tilde{\xi}}{\partial z}+\tilde{w} \frac{\partial \rho^{2}}{\partial z} \\
& \quad=\frac{1}{F r} \tilde{w}, \quad \text { in } L^{2}\left([0, T] ; H^{-1}\left(\mathbb{T}^{3}\right)\right) .
\end{aligned}
$$

Clearly, we can take the inner product of (3.33a) with $\tilde{\mathbf{u}}_{h},(3.33 \mathrm{~b})$ with $\tilde{w}$, (3.33c) with $<\tilde{\rho}>_{z}$ and $(3.33 \mathrm{~d})$ with $\tilde{\xi}$. Using the divergence free condition, $\nabla_{h} \cdot \mathbf{u}_{h}^{1}=\nabla_{h} \cdot \mathbf{u}_{h}^{2}=\nabla_{h} \cdot \tilde{\mathbf{u}}_{h}=0, \frac{\partial w^{1}}{\partial z}=\frac{\partial w^{2}}{\partial z}=\frac{\partial \tilde{w}}{\partial z}=0$, integration by parts, Hölder inequality and Young's inequality, we can show that

$$
\begin{aligned}
& \frac{1}{2} \frac{d}{d t}\left\|\tilde{\mathbf{u}}_{h}\right\|_{L_{h}^{2}\left(\mathbb{T}^{2}\right)}^{2} \leq\left|\left(\left(\tilde{\mathbf{u}}_{h} \cdot \nabla_{h}\right) \tilde{\mathbf{u}}_{h}^{2}, \tilde{\mathbf{u}}_{h}\right)_{h}\right| \\
& \leq\left\|\nabla_{h} \mathbf{u}_{h}^{2}\right\|_{L_{h}^{\infty}\left(\mathbb{T}^{2}\right)}\left\|\tilde{\mathbf{u}}_{h}\right\|_{L_{h}^{2}\left(\mathbb{T}^{2}\right)}^{2}, \\
& \frac{1}{2} \frac{d}{d t}\left(\|\tilde{w}\|_{L_{h}^{2}\left(\mathbb{T}^{2}\right)}^{2}+\left\|\langle\tilde{\rho}\rangle_{z}\right\|_{L_{h}^{2}\left(\mathbb{T}^{2}\right)}^{2}\right) \\
& \leq\left|\left(\left(\tilde{\mathbf{u}}_{h} \cdot \nabla_{h}\right) w^{2}, \tilde{w}\right)_{h}\right|+\left|\left(\left(\tilde{\mathbf{u}}_{h} \cdot \nabla_{h}\right)\left\langle\rho^{2}\right\rangle_{z},\langle\tilde{\rho}\rangle_{z}\right)_{h}\right| \\
& \leq\left\|\tilde{\mathbf{u}}_{h}\right\|_{L_{h}^{2}\left(\mathbb{T}^{2}\right)}\left(\left\|\nabla_{h} w^{2}\right\|_{L_{h}^{\infty}\left(\mathbb{T}^{2}\right)}\|\tilde{w}\|_{L_{h}^{2}\left(\mathbb{T}^{2}\right)}\right. \\
&\left.\quad+\left\|\nabla_{h}\left\langle\rho^{2}\right\rangle_{z}\right\|_{L_{h}^{\infty}\left(\mathbb{T}^{2}\right)}\left\|\langle\tilde{\rho}\rangle_{z}\right\|_{L_{h}^{2}\left(\mathbb{T}^{2}\right)}\right) \\
& \leq\left(\left\|\nabla_{h} w^{2}\right\|_{L_{h}^{\infty}\left(\mathbb{T}^{2}\right)}+\left\|\nabla_{h}\left\langle\rho^{2}\right\rangle_{z}\right\|_{L_{h}^{\infty}\left(\mathbb{T}^{2}\right)}\right) D(t)
\end{aligned}
$$

and

$$
\begin{aligned}
\frac{1}{2} \frac{d}{d t}\|\nabla \tilde{\xi}\|_{L^{2}\left(\mathbb{T}^{3}\right)}^{2} \leq & \left|\left(\left(\mathbf{u}_{h}^{1} \cdot \nabla_{h}\right) \tilde{\xi}, \Delta \tilde{\xi}\right)\right|+\left|\left(\left(\tilde{\mathbf{u}}_{h} \cdot \nabla_{h}\right) \tilde{\xi}, \Delta \xi^{2}\right)\right| \\
& +\left|\left(w^{1} \frac{\partial \tilde{\xi}}{\partial z}, \Delta \tilde{\xi}\right)\right|+\left|\left(\tilde{w} \frac{\partial \tilde{\xi}}{\partial z}, \rho^{2}\right)\right|+\frac{1}{F r}|(\tilde{w}, \tilde{\xi})| \\
\leq & \sum_{j=1}^{2}\left|\left(\frac{\partial \mathbf{u}_{h}^{1}}{\partial x_{j}}, \nabla_{h} \tilde{\xi} \frac{\partial \tilde{\xi}}{\partial x_{j}}\right)\right|+\left|\left(\left(\tilde{\mathbf{u}}_{h} \cdot \nabla_{h}\right) \tilde{\xi}, \Delta \xi^{2}\right)\right|
\end{aligned}
$$




$$
\begin{aligned}
& \quad+\left|\left(\nabla_{h} w^{1} \frac{\partial \tilde{\xi}}{\partial z}, \nabla_{h} \tilde{\xi}\right)\right|+\left|\left(\tilde{w} \frac{\partial \tilde{\xi}}{\partial z}, \rho^{2}\right)\right|+\frac{1}{F r}|(\tilde{w}, \tilde{\xi})| \\
& \leq\left\|\nabla_{h} \mathbf{u}_{h}^{1}\right\|_{L^{\infty}\left(\mathbb{T}^{3}\right)}\left\|\nabla_{h} \tilde{\xi}\right\|_{L^{2}\left(\mathbb{T}^{3}\right)}^{2} \\
& +\left\|\tilde{\mathbf{u}}_{h}\right\|_{L^{2}\left(\mathbb{T}^{3}\right)}\left\|\nabla_{h} \tilde{\xi}\right\|_{L^{2}\left(\mathbb{T}^{3}\right)}\left\|\Delta \xi^{2}\right\|_{L^{\infty}\left(\mathbb{T}^{3}\right)} \\
& +\left\|\nabla_{h} w^{1}\right\|_{L^{\infty}\left(\mathbb{T}^{3}\right)}\|\nabla \tilde{\xi}\|_{L^{2}\left(\mathbb{T}^{3}\right)}^{2} \\
& +\left\|\rho^{2}\right\|_{L^{\infty}\left(\mathbb{T}^{3}\right)}\|\tilde{w}\|_{L^{2}\left(\mathbb{T}^{3}\right)}\|\nabla \tilde{\xi}\|_{L^{2}\left(\mathbb{T}^{3}\right)} \\
& +\frac{1}{F r}\|\tilde{w}\|_{L^{2}\left(\mathbb{T}^{3}\right)}\|\tilde{\xi}\|_{L^{2}\left(\mathbb{T}^{3}\right)} .
\end{aligned}
$$

Using Poincaré inequality, Lemma 2.2, we may conclude that

$$
\begin{aligned}
\frac{1}{2} \frac{d}{d t}\|\nabla \tilde{\xi}\|_{L^{2}\left(\mathbb{T}^{3}\right)}^{2} \leq & C(L, F r)\left(\left\|\nabla_{h} \mathbf{u}_{h}^{1}\right\|_{L_{h}^{\infty}\left(\mathbb{T}^{2}\right)}+\left\|\rho^{2}\right\|_{L^{\infty}\left(\mathbb{T}^{3}\right)}\right. \\
& \left.+\left\|\nabla_{h} w^{1}\right\|_{L_{h}^{\infty}\left(\mathbb{T}^{2}\right)}\right) D(t),
\end{aligned}
$$

where $C(L, F r)$ is a constant that depends on the size of the domain $L$ and the Froude number $F r$. Recall that by assumption, $\mathbf{u}_{h}^{1}, \rho^{2}$ and $w^{1}$ are strong solutions in the sense of Definition 3.5, and they satisfy the estimates in Proposition 3.1 and Proposition 3.2 with corresponding initial data $\mathbf{u}_{h}^{1,0}$, $\rho^{2,0}$ and $w^{1,0}$, respectively. Thus, adding (3.34), (3.35) and (3.36) imply that

$$
\frac{d}{d t} D(t) \leq C_{0}^{1,2}(T, L, F r) D(t)
$$

where $C_{0}^{1,2}(T, L, F r)$ is a constant that depends on $T, L, F r$ and the norms of the initial data $\mathbf{u}_{h}^{1,0}, w^{1,0}$ and $\rho^{2,0}$. Integrating the above inequality on the time interval $[0, t]$, for $t \leq T$, proves (3.32) and completes the proof.

\section{Global existence and uniqueness of weak solutions using vorticiy formulation}

In this section, we aim to prove the global existence and uniqueness of weak solutions of the inviscid slow-limiting dynamics model in vorticity formulation. We define the vorticity $\omega=\nabla_{h} \times \mathbf{u}_{h}$. As in the case of the two-dimensional incompressible Euler equations in vorticity formulation, we have the analogue of the two-dimensional periodic Biot-Savart law (2.6). 
We explicitly restrict ourselves to the unique solution $\mathbf{u}_{h}$ of the elliptic system: $\nabla_{h} \times \mathbf{u}_{h}=\omega$ and $\nabla_{h} \cdot \mathbf{u}_{h}=0$, that satisfies the side condition $\int_{\mathbb{T}^{2}} \mathbf{u}_{h}(x, y) d x d y=0$.

Since we are considering periodic boundary conditions, we can write

$$
\rho(\mathbf{x})=\sum_{k=-\infty}^{\infty} \rho_{k}\left(\mathbf{x}_{h}\right) e^{\frac{2 \pi}{L} i k z}, \quad \text { where } \quad \rho_{k}\left(\mathbf{x}_{h}\right)=\frac{1}{L} \int_{0}^{L} \rho(\mathbf{x}) e^{-\frac{2 \pi}{L} i k z} d z
$$

are periodic in $\mathbb{T}^{2}$, for each $k \in \mathbb{Z}$. Notice that $\rho_{0}\left(\mathbf{x}_{h}\right) \equiv\langle\rho\rangle_{z}\left(\mathbf{x}_{h}\right)$. We may take the horizontal curl of equation (1.3a) and take the Fourier transform of equation (1.3d) and rewrite system (1.3) in vorticity-Fourier transform formulation as

$$
\begin{aligned}
& \frac{\partial \omega}{\partial t}+\left(\mathbf{u}_{h} \cdot \nabla_{h}\right) \omega=0 \\
& \frac{\partial w}{\partial t}+\left(\mathbf{u}_{h} \cdot \nabla_{h}\right) w=-\frac{1}{F r}\langle\rho\rangle_{z}, \\
& \omega\left(0 ; \mathbf{x}_{h}\right)=\omega^{0}\left(\mathbf{x}_{h}\right), \quad w\left(0 ; \mathbf{x}_{h}\right)=w^{0}\left(\mathbf{x}_{h}\right), \quad\langle\rho\rangle_{z}\left(0 ; \mathbf{x}_{h}\right)=\left\langle\rho^{0}\right\rangle_{z}\left(\mathbf{x}_{h}\right),
\end{aligned}
$$

and

(4.1g) $\quad \rho_{k}\left(0 ; \mathbf{x}_{h}\right)=\rho_{k}^{0}\left(\mathbf{x}_{h}\right), \quad$ where $\quad \rho_{k}^{0}\left(\mathbf{x}_{h}\right)=\frac{1}{L} \int_{0}^{L} \rho^{0}(\mathbf{x}) e^{-\frac{2 \pi}{L} i k z} d z$

for each $k \in \mathbb{Z} \backslash\{0\}$.

Definition 4.1 (Weak Solutions). For any $1<q \leq \infty$, let $\omega^{0}\left(\mathbf{x}_{h}\right) \in L_{h}^{\infty}\left(\mathbb{T}^{2}\right)$, $w^{0}\left(\mathbf{x}_{h}\right) \in L_{h}^{\infty}\left(\mathbb{T}^{2}\right),\left\langle\rho^{0}\right\rangle_{z}\left(\mathbf{x}_{h}\right) \in L_{h}^{\infty}\left(\mathbb{T}^{2}\right)$ and $\rho_{k}^{0}\left(\mathbf{x}_{h}\right) \in L_{h}^{q}\left(\mathbb{T}^{2}\right)$, for each $k \in \mathbb{Z} \backslash\{0\}$. For any $T>0$, we say that $\omega\left(t ; \mathbf{x}_{h}\right), w\left(t ; \mathbf{x}_{h}\right)$ and $\rho(t ; \mathbf{x})=$ $\sum_{k=-\infty}^{\infty} \rho_{k}\left(\mathbf{x}_{h}\right) e^{\frac{2 \pi}{L} i k z}$ is a weak solution of system (4.1), on the time interval $[0, T]$, if

$$
\begin{aligned}
\omega & \in L^{\infty}\left(\left[0, T\left[; L_{h}^{\infty}\left(\mathbb{T}^{2}\right)\right),\right.\right. \\
w & \in L^{\infty}\left([0, T] ; L_{h}^{\infty}\left(\mathbb{T}^{2}\right)\right), \\
\langle\rho\rangle_{z} & \in L^{\infty}\left([0, T] ; L_{h}^{\infty}\left(\mathbb{T}^{2}\right)\right),
\end{aligned}
$$




$$
\rho_{k} \in L^{\infty}\left([0, T] ; L_{h}^{q}\left(\mathbb{T}^{2}\right)\right),
$$

for each $k \in \mathbb{Z} \backslash\{0\}$. Moreover, $\omega, w$ and $\rho_{k}$ satisfy (4.1) in the distribution sense for each $k$; that is, for any $\phi\left(t ; \mathbf{x}_{h}\right), \chi_{1}\left(t ; \mathbf{x}_{h}\right), \chi_{2}\left(t ; \mathbf{x}_{h}\right) \in \mathcal{D}\left([0, T] \times \mathbb{T}^{2}\right)$, with $\phi\left(T ; \mathbf{x}_{h}\right)=\chi_{1}\left(T ; \mathbf{x}_{h}\right)=\chi_{2}\left(T ; \mathbf{x}_{h}\right)=0$, and any $\psi\left(t ; \mathbf{x}_{h}\right) \in \mathcal{D}([0, T] \times$ $\left.\mathbb{T}^{2}\right)$ with $\psi\left(T ; \mathbf{x}_{h}\right)=0$, such that $\nabla_{h} \cdot \phi=\nabla_{h} \cdot \chi_{1}=\nabla_{h} \cdot \chi_{2}=\nabla_{h} \cdot \psi=0$, the integral identities $(3.25 \mathrm{~b}),(3.25 \mathrm{c})$ and

$$
\begin{aligned}
& \int_{0}^{T}\left(\omega(s), \phi^{\prime}(s)\right)_{h} d s+\int_{0}^{T}\left(\left(\mathbf{u}_{h}(s) \cdot \nabla_{h}\right) \phi(s), \omega(s)\right)_{h} d s \\
& \quad=-\left(\mathbf{u}_{h}^{0}, \phi^{0}\right)_{h}, \\
& \int_{0}^{T}\left(\rho_{k}(s), \psi^{\prime}(s)\right)_{h} d s+\int_{0}^{T}\left(\left(\mathbf{u}_{h}(s) \cdot \nabla_{h}\right) \psi(s), \rho_{k}(s)\right)_{h} d s \\
& \quad=i k \int_{0}^{T}\left(w(s) \rho_{k}(s), \psi(s)\right)_{h} d s-\left(\rho_{k}^{0}, \psi^{0}\right)_{h},
\end{aligned}
$$

hold for each $k \in \mathbb{Z} \backslash\{0\}$.

Theorem 4.2 (Global existence and uniqueness of weak solutions). For any $1<q \leq \infty$, let $\omega^{0} \in L_{h}^{\infty}\left(\mathbb{T}^{2}\right), w^{0} \in L_{h}^{\infty}\left(\mathbb{T}^{2}\right),\left\langle\rho^{0}\right\rangle_{z} \in L_{h}^{\infty}\left(\mathbb{T}^{2}\right)$ and $\rho_{k}^{0} \in L_{h}^{q}\left(\mathbb{T}^{2}\right)$, for each $k \in \mathbb{Z} \backslash\{0\}$. Then, for any given $T>0$, system (4.1) has a unique weak solution in the sense of Definition 4.1.

Proof. Given $\omega^{0}\left(\mathbf{x}_{h}\right) \in L_{h}^{\infty}\left(\mathbb{T}^{2}\right)$ and $T>0$, by Theorem 2.3, the twodimensional incompressible Euler equations in vorticity formulation have a unique solution $\omega\left(t ; \mathbf{x}_{h}\right) \in L^{\infty}\left([0, T] ; L_{h}^{\infty}\left(\mathbb{T}^{2}\right)\right)$ such that

$$
\|\omega(t)\|_{L_{h}^{p}\left(\mathbb{T}^{2}\right)}=\left\|\omega^{0}\right\|_{L_{h}^{p}\left(\mathbb{T}^{2}\right)},
$$

for any $p \in[1, \infty]$. Moreover, by the elliptic regularity estimate (2.1), $\mathbf{u}_{h}\left(t ; \mathbf{x}_{h}\right) \in L^{\infty}\left([0, T] ; W_{h}^{1, p}\left(\mathbb{T}^{2}\right)\right)$ for any $p \in[1, \infty)$ and

$$
\left\|\mathbf{u}_{h}(t)\right\|_{W_{h}^{1, p}\left(\mathbb{T}^{2}\right)} \leq C p\left\|\omega^{0}\right\|_{L_{h}^{\infty}\left(\mathbb{T}^{2}\right)},
$$

for all $t \in[0, T]$. The existence and uniqueness of a weak solution $w\left(t ; \mathbf{x}_{h}\right) \in$ $L^{\infty}\left([0, T] ; L_{h}^{\infty}\left(\mathbb{T}^{2}\right)\right)$ and $\langle\rho\rangle_{z}\left(t ; \mathbf{x}_{h}\right) \in L^{\infty}\left([0, T] ; L_{h}^{\infty}\left(\mathbb{T}^{2}\right)\right)$ that satisfies the inviscid system (3.26) in the weak sense, and the estimate (3.3), is presented in the proof of Theorem 3.4.

Since $\mathbf{u}_{h}, w \in L^{\infty}\left([0, T] ; L_{h}^{\infty}\left(\mathbb{T}^{2}\right)\right)$ and $\nabla_{h} \cdot \mathbf{u}_{h}=0$, then the existence of a solution $\rho_{k}\left(t ; \mathbf{x}_{h}\right) \in L^{\infty}\left([0, T] ; L_{h}^{q}\left(\mathbb{T}^{2}\right)\right)$ of $(4.1 \mathrm{~d})$, for each $k \in \mathbb{Z} \backslash\{0\}$, will follow directly by Theorem 2.5. The uniqueness of the solution $\rho_{k}\left(t ; \mathbf{x}_{h}\right)$, for 
each $k \in \mathbb{Z} \backslash\{0\}$, follows by Theorem 2.6 since $\mathbf{u}_{h} \in L^{\infty}\left([0, T] ; W_{h}^{1, p}\left(\mathbb{T}^{2}\right)\right)$ for any $p \in[1, \infty)$. This completes the proof.

\section{Acknowledgements}

This paper is dedicated to Professor Marshall Slemrod on the occasion of his $70^{\text {th }}$ birthday as a token of friendship and admiration. The work of C.C. is supported in part by the NSF grant DMS-1109022. The work of E.S.T. is supported in part by the NSF grants DMS-1009950, DMS-1109640 and DMS-1109645, and by the Minerva Stiftung/Foundation.

\section{References}

[1] C. Bardos, Existence et unicité de la solution de l'équation d'Euler en dimension deux, J. Math. Anal. Appl. 40 (1972), 769-790. MR0333488

[2] C. Bardos and E. S. Titi, Euler equations for incompressible ideal fluids, Uspekhi Matematicheskikh Nauk, UMN 62:3(375) (2007), 5-46. Also in Russian Mathematical Surveys 62(3) (2007), 409-451. MR2355417

[3] C. Bardos and E. S. Titi, Loss of smoothness and energy conserving rough weak solutions for the $3 d$ Euler equations, Discrete Contin. Dyn. Syst. Ser. S 3(2) (2010), 185-197. MR2610558

[4] C. Bardos and E. S. Titi, Mathematics and turbulence: where do we stand? Journal of Turbulence 14(3) (2013), 42-76. MR3174319

[5] J. T. Beale, T. Kato, and A. Majda, Remarks on the Breakdown of Smooth Solutions for the 3-D Euler Equations, Commun. Math. Phys. 94 (1984), 61-66. MR0763762

[6] P. Constantin and C. Foias, Navier-Stokes Equations, Chicago Lectures in Mathematics, University of Chicago Press, Chicago, IL, 1988. MR0972259

[7] P. Constantin, On the Euler equations of incompressible fluids, Bull. Amer. Math. Soc. 44 (2007), 603-621. MR2338368

[8] R.J. DiPerna and P.L. Lions, Ordinary differential equations, transport theory and Sobolev spaces, Invent. Math. 98 (1989), 511-547. MR1022305

[9] L. C. Evans, Partial Differential Equations, Graduate Studies in Mathematics, vol. 19, AMS, Providence, RT, 1998. MR1625845 (99e:35001) 
[10] T. Kato, Remarks on the Euler and Navier-Stokes equations in $\mathbb{R}^{2}$, Proc. Symp. Pure Math., 45, Amer. Math. Soc., Providence, (1986), 1-7. MR0843590

[11] T. Kato and G. Ponce, Well-posedness of the Euler and Navier-Stokes equations in the Lebesgue spaces $L_{s}^{p}\left(\mathbb{R}^{2}\right)$, Rev. Mat. Iberoamericana 2(1-2) (1986), 73-88. MR0864654

[12] T. Kato, A remark on a theorem of C. Bardos on the 2D-Euler equation, (U. C. Berkeley Pre-print), 1990.

[13] A. Larios, E. Lunasin, and E. S. Titi, Global well-posedness for the 2D Boussinesq system with anisotropic viscosity and without heat diffusion, J. Differential Equations 255 (2013) 2636-2654. arXiv:1010.5024v1. MR3090072

[14] L. Lichtenstein, Über einige Existenzprobleme der Hydrodynamik homogener, unzusammendrückbarer, reibungsloser Flüssigkeiten und die Helmholtzschen Wirbelsätze, Math. Z. 23(1) (1925), 89-154. MR1544733

[15] A. J. Majda and A. L. Bertozzi, Vorticity and Incompressible Flow, Cambridge Texts in Applied Mathematics, vol. 27, Cambridge University Press, Cambridge, 2002. MR1867882 (2003a:76002)

[16] J. Pedlosky, Geophysical Fluid Dynamics, Springer-Verlag, New York, 1987.

[17] B. A. Wingate, P. Embid, M. Holmes-Cerfon, and M. A. Taylor, Low Rossby limiting dynamics for stably stratified flow with finite Froude number, J. Fluid Mech. 676 (2011), 546-571. MR2804461

[18] V. I. Yudovich, Non-stationary flow of an ideal incompressible liquid, Zh. Vych. Mat. 3(6) (1963), 1032-1066 (English).

Chongsheng Cao

Department of Mathematics

FLORIDA INTERNATIONAL UNIVERSITY

MiAMI, FL 33199

USA

E-mail address: caoc@fiu.edu

Aseel Farhat

Department of Mathematics

INDIANA UNIVERSITY BLOOMINGTON

BLOOMINGTON, IN 47405

USA

E-mail address: afarhat@indiana.edu 
EDRISS S. TITI

Department of Mathematics

AND

Department of Mechanical and Aero-Space Engineering

UNIVERSITY OF CALIFORNIA

IRVINE, CA 92697

$\mathrm{USA}$

E-mail address: etiti@math.uci.edu

Also

The Department of Computer Science and Applied Mathematics The Weizmann Institute of Science

REHOVOT 76100

ISRAEL

E-mail address: edriss.titi@weizmann.ac.il

Received November 23, 2013 